\title{
On Kolmogorov equations for anisotropic multivariate Levy processes
}

\section{Journal Article}

\section{Author(s):}

Reich, N.; Schwab, Christoph; Winter, C.

Publication date:

2010-12

Permanent link:

https://doi.org/10.3929/ethz-b-000027293

Rights / license:

In Copyright - Non-Commercial Use Permitted

Originally published in:

Finance and Stochastics 14(4), https://doi.org/10.1007/s00780-009-0108-x 


\title{
On Kolmogorov equations for anisotropic multivariate Lévy processes
}

\author{
N. Reich • C. Schwab • C. Winter
}

Received: 9 April 2008 / Accepted: 3 December 2008 / Published online: 17 December 2009

(C) Springer-Verlag 2009

\begin{abstract}
For $d$-dimensional exponential Lévy models, variational formulations of the Kolmogorov equations arising in asset pricing are derived. Well-posedness of these equations is verified. Particular attention is paid to pure jump, $d$-variate Lévy processes built from parametric, copula dependence models in their jump structure. The domains of the associated Dirichlet forms are shown to be certain anisotropic Sobolev spaces. Singularity-free representations of the Dirichlet forms are given which remain bounded for piecewise polynomial, continuous functions of finite element type. We prove that the variational problem can be localized to a bounded domain with explicit localization error bounds. Furthermore, we collect several analytical tools for further numerical analysis.
\end{abstract}

Keywords Lévy copulas · Lévy processes · Integro-differential equations · Pseudo-differential operators $\cdot$ Dirichlet forms $\cdot$ Option pricing

Mathematics Subject Classification (2000) $45 \mathrm{~K} 05 \cdot 60 \mathrm{~J} 75 \cdot 65 \mathrm{M} 60$

\section{JEL Classification $\mathrm{C} 02$}

\section{Introduction}

Consider a basket of $d \geq 1$ risky assets whose log returns $X_{t}$ at time $t>0$ are modeled by a Lévy process $\bar{X}=\left\{X_{t}\right\}_{t \geq 0}$ with state space $\mathbb{R}^{d}$. By the fundamental theorem of asset pricing [17], arbitrage-free prices $u$ of European contingent claims on such baskets with "reasonable" payoffs $g(\cdot)$ and maturity $T$ are given by the conditional

N. Reich · C. Schwab $(\bowtie) \cdot$ C. Winter

Seminar for Applied Mathematics, ETH Zürich, 8092 Zürich, Switzerland

e-mail: schwab@math.ethz.ch 
expectation

$$
u(t, x)=\mathbb{E}\left(e^{-r(T-t)} g\left(X_{T}\right) \mid X_{t}=x\right) .
$$

Here, the expectation is taken with respect to an a priori chosen martingale measure equivalent to the historical measure (see, e.g., $[18,19]$ for some measure selection criteria).

It is well known that the family $\left\{T_{t}\right\}_{t \geq 0}$ of maps $T_{t}: g(\cdot) \mapsto u(t, \cdot)$ is a oneparameter semigroup. We denote by $\mathcal{A}$ its associated infinitesimal generator, i.e.,

$$
\mathcal{A} u:=\lim _{t \rightarrow 0+} \frac{1}{t}\left(T_{t} u-u\right)
$$

for all functions $u \in \mathcal{D}(\mathcal{A})$ in the domain

$$
\mathcal{D}(\mathcal{A}):=\left\{u \in C_{\infty}\left(\mathbb{R}^{d}\right): \lim _{t \rightarrow 0+} \frac{1}{t}\left(T_{t} u-u\right) \text { exists as strong limit }\right\}
$$

where $C_{\infty}\left(\mathbb{R}^{d}\right)$ is the space of continuous functions vanishing at infinity (see, e.g., [22]). Sufficiently smooth value functions $u$ in (1.1) can be obtained as classical solutions of a partial integro-differential equation (PIDE), the Kolmogorov equation

$$
\frac{\partial u}{\partial t}+\mathcal{A} u-r u=0
$$

where $\mathcal{A}$ is the infinitesimal generator of the process $X$ defined by (1.2). Among several possible notions of solution (classical, variational, and viscosity solutions, to name the most frequently employed), we opt for variational solutions which are the basis for variational discretization methods such as finite element discretizations. To convert (1.3) into variational form, we formally integrate against a test function $v$ and obtain (assuming $r=0$ for convenience)

$$
\frac{d}{d t}(u, v)+\underbrace{\mathcal{E}(u, v)}_{(\mathcal{A} u, v)}=0 .
$$

Here, the bilinear expression $\mathcal{E}(u, v)$ denotes the extension of the $L^{2}\left(\mathbb{R}^{d}\right)$ inner product $(\mathcal{A} u, v)$ corresponding to $X$ from $u, v \in C_{0}^{\infty}\left(\mathbb{R}^{d}\right)$ by continuity to the domain $\mathcal{D}(\mathcal{E})$. For the class of Lévy processes considered, we show in this paper that $\mathcal{E}(\cdot, \cdot)$ is in fact a Dirichlet form.

In the univariate case, i.e., for a Lévy process $X$ with state space $\mathbb{R},(1.3),(1.4)$ and methods for their numerical solution have been studied by several authors, see, e.g., [7, 12, 28, 29] and the references therein. The numerical methods investigated were either finite difference methods [7, 12] approximating viscosity solutions or variational methods [28, 29] approximating weak (or variational) solutions. Both solution concepts coincide for sufficiently smooth solutions, but the resulting numerical schemes have essentially different properties. In [20], the univariate variational setting was extended to $d>1$ dimensions for pure jump processes built from 
1-homogeneous Lévy copulas and univariate marginal Lévy processes with symmetric tempered stable margins. The domain of the infinitesimal generator $\mathcal{A}$ was characterized, and it was shown that the corresponding variational problem is well posed. Under these processes, option pricing using Fourier methods as in [8] is generally not possible since the characteristic functions are not given in closed form.

The goal of this work is twofold. First, we extend [20] to the multivariate, nonsymmetric case, i.e., where the univariate marginal Lévy processes are tempered stable but with possibly nonsymmetric margins. Second, we provide further analytical results that are required for an efficient numerical implementation of (1.4). We show that in the pure jump case the domain $\mathcal{D}(\mathcal{E})$ of the Dirichlet form $\mathcal{E}(\cdot, \cdot)$ of $X$ belongs to a certain class of anisotropic Sobolev spaces and $\mathcal{E}(\cdot, \cdot)$ satisfies a Gårding inequality on these spaces. In addition, $\mathcal{E}(\cdot, \cdot)$ is cast into several forms which are equivalent on $C_{0}^{\infty}\left(\mathbb{R}^{d}\right)$ and which are well defined for piecewise polynomial, globally Lipschitz-continuous arguments. We show that these forms naturally compensate the singularity of the jump measure near zero arising from the square-summable small jumps. There is no need to approximate the small jumps by a Brownian motion. These reformulations apply for any Lévy process with state space $\mathbb{R}^{d}$ and are the basis for a variational discretization of (1.3) by, e.g., finite element methods. Furthermore, we derive the pricing PIDEs for $d$-dimensional Lévy models and obtain the corresponding variational formulation with explicit Sobolev characterization of the ansatz and test spaces. Extending [20], we establish sufficient conditions on $X$ to render the bilinear form $\mathcal{E}(\cdot, \cdot)$ a nonsymmetric Dirichlet form in the sense of Berg and Forst [3]. We deduce the existence of a unique solution to the variational formulation of the problem for a class of copulas and nonsymmetric marginal processes. To allow the implementation of the variational problem, we furthermore localize it to the bounded domain $G_{R}=[-R, R]^{d}$ and show that the solution of the localized problem converges pointwise exponentially in $R$ to the exact solution of the original problem. We briefly describe the finite element discretization and study numerically the quantitative effect on option prices of the diffusion approximation of small jumps proposed, e.g., in $[12,13]$.

Throughout this work, we write $x \lesssim y$ to express that $x$ is bounded by a constant multiple of $y$. For $\mathcal{B} \subset \mathbb{R}^{d}$, by $1_{\mathcal{B}}: \mathbb{R}^{d} \rightarrow\{0,1\}$ we denote the indicator function of the set $\mathcal{B}$.

\section{Preliminaries}

We recapitulate several tools needed subsequently. First, we present some classical facts on Lévy processes and their generators and describe a class of parametric copula constructions for dependence in jumps of multivariate Lévy processes. Finally, we collect some abstract results on variational parabolic evolution and inequality problems.

\subsection{Lévy processes}

A càdlàg stochastic process $X=\left\{X_{t}\right\}_{t \geq 0}$ with state space $\mathbb{R}^{d}$ such that $X_{0}=0$ a.s. is called a Lévy process if it has independent and stationary increments and is stochas- 
tically continuous. The characteristic exponent $\psi: \mathbb{R}^{d} \rightarrow \mathbb{C}$ of $X$ is defined by

$$
\mathbb{E}\left(e^{i\left\langle\xi, X_{t}\right\rangle}\right)=e^{-t \psi(\xi)}, \quad \xi \in \mathbb{R}^{d}, t \geq 0 .
$$

It is a continuous, negative definite function for which we have the Lévy-Khinchin representation (cf., e.g., [38, Theorem 8.1] or [22])

$$
\psi(\xi)=-i\langle\gamma, \xi\rangle+\frac{1}{2}\langle\xi, \mathcal{Q} \xi\rangle+\int_{\mathbb{R}^{d}}\left(1-e^{i\langle\xi, z\rangle}+i\langle\xi, z\rangle 1_{\{|z| \leq 1\}}\right) v(\mathrm{~d} z),
$$

where $\mathcal{Q} \in \mathbb{R}_{\text {sym }}^{d \times d}$ denotes the covariance matrix of the continuous part of $X, \gamma \in \mathbb{R}^{d}$ the drift of $X$, and $v$ is the Lévy measure which satisfies

$$
\int_{\mathbb{R}^{d}}\left(1 \wedge|z|^{2}\right) v(\mathrm{~d} z)<\infty
$$

The triplet $(\mathcal{Q}, v, \gamma)$ is called characteristic triplet of the process $X$.

No arbitrage considerations require Lévy processes employed in mathematical finance to be martingales. The following result gives conditions on the characteristic triplet which ensure this.

Lemma 2.1 Let $X=\left(X^{1}, \ldots, X^{d}\right)^{\top} \in \mathbb{R}^{d}$ be a Lévy process with characteristic triplet $(\mathcal{Q}, v, \gamma)$. Assume $\int_{|z|>1} e^{z_{j}} v(\mathrm{~d} z)<\infty, j=1, \ldots, d$. Then $e^{X^{j}}$ is a martingale with respect to the canonical filtration $\mathcal{F}$ of $X$ if and only if

$$
\frac{\mathcal{Q}_{j j}}{2}+\gamma_{j}+\int_{\mathbb{R}^{d}}\left(e^{z_{j}}-1-z_{j} 1_{\{|z| \leq 1\}}\right) v(\mathrm{~d} z)=0 .
$$

Proof It is shown in [32, (2.30)] that Lemma 2.1 holds for general semimartingales and therefore in particular for Lévy processes.

Based on $\psi(\xi)$ in (2.1), it is well known that the infinitesimal generator $\mathcal{A}$ in (1.2) corresponding to the Lévy process $X$ is a pseudo-differential operator acting on $u \in C_{0}^{\infty}\left(\mathbb{R}^{d}\right)$ by the (oscillatory) integral

$$
(\mathcal{A} u)(x)=(\psi(D) u)(x)=(2 \pi)^{-d} \int_{\mathbb{R}^{d}} e^{i\langle\xi, x\rangle} \psi(\xi) \hat{u}(\xi) \mathrm{d} \xi,
$$

where $\hat{u}(\xi):=(2 \pi)^{-d} \int e^{-i\langle\xi, z\rangle} u(z) \mathrm{d} z$ denotes the Fourier transform of $u$.

\subsection{Lévy copulas}

Since the law of a Lévy process $X$ is time-homogeneous, it is completely characterized by its characteristic triplet $(\mathcal{Q}, v, \gamma)$. The drift $\gamma$ has no effect on the dependence structure between components of $X$. The dependence structure of the Brownian motion part of $X$ is given by its covariance matrix $\mathcal{Q}$. For the purposes of financial modeling, it remains to specify a parametric dependence structure of the purely discontinuous part of $X$ which can be done using Lévy copulas. Lévy copulas have 
been introduced first by Tankov [43] and were further developed by Kallsen and Tankov [25]. We refer to [25] for an introduction to Lévy copulas and just state one of the main results, Sklar's theorem for Lévy copulas. For this, we need to introduce tail integrals of Lévy processes.

Definition 2.2 Let $X$ be a Lévy process with state space $\mathbb{R}^{d}$ and Lévy measure $v$. The tail integral of $X$ is the function $U: \mathbb{R}^{d} \backslash\{0\} \rightarrow \mathbb{R}$ given by

$$
U\left(x_{1}, \ldots, x_{d}\right)=\prod_{j=1}^{d} \operatorname{sgn}\left(x_{j}\right) v\left(\prod_{j=1}^{d} \mathcal{I}\left(x_{j}\right)\right),
$$

where

$$
\mathcal{I}(x)= \begin{cases}(x, \infty), & \text { for } x \geq 0, \\ (-\infty, x], & \text { for } x<0 .\end{cases}
$$

Furthermore, for $I \subset\{1, \ldots, d\}$ nonempty, the $I$-marginal tail integral $U^{I}$ of $X$ is the tail integral of the process $X^{I}:=\left(X_{i}\right)_{i \in I}$. If $I=\{i\}$, we write $U_{i}=U^{\{i\}}$. We also use the notation $x^{I}:=\left(x_{i}\right)_{i \in I}$ and for $x \in \mathbb{R}^{d}, y \in \mathbb{R}^{|I|}$,

$$
x+y^{I}=z \in \mathbb{R}^{d} \quad \text { with } z_{i}= \begin{cases}x_{i}, & \text { if } i \notin I, \\ x_{i}+y_{i}, & \text { else. }\end{cases}
$$

The next result [25, Theorem 3.6] shows that essentially any Lévy process $X$ with values in $\mathbb{R}^{d}$ can be built from univariate marginal processes $X_{i}$ and Lévy copulas.

Theorem 2.3 (Sklar's theorem for Lévy copulas) For any Lévy process $X$ with state space $\mathbb{R}^{d}$, there exists a Lévy copula $F$ such that the tail integrals of $X$ satisfy

$$
U^{I}\left(x^{I}\right)=F^{I}\left(\left(U_{i}\left(x_{i}\right)\right)_{i \in I}\right)
$$

for any nonempty $I \subset\{1, \ldots, d\}$ and any $\left(x_{i}\right)_{i \in I} \in \mathbb{R}^{|I|} \backslash\{0\}$. The Lévy copula $F$ is unique on $\prod_{i=1}^{d} \overline{\text { Range } U_{i}}$.

Conversely, let $F$ be a d-dimensional Lévy copula and $U_{i}, i=1, \ldots, d$, tail integrals of univariate Lévy processes. Then, there exists a d-dimensional Lévy process $X$ such that its components have tail integrals $U_{i}$ and its marginal tail integrals satisfy (2.3). The Lévy measure $v$ of $X$ is uniquely determined by $F$ and $U_{i}, i=1, \ldots, d$.

Lévy copulas $F$ allow parametric constructions of multivariate jump densities from univariate ones.

Remark 2.4 Let $U_{1}, \ldots, U_{d}$ be one-dimensional tail integrals with Lévy densities $k_{1}, \ldots, k_{d}$, and $F$ a Lévy copula such that $\partial_{1} \cdots \partial_{d} F$ exists in the sense of distributions. Then

$$
k\left(x_{1}, \ldots, x_{d}\right)=\left.\partial_{1} \cdots \partial_{d} F\right|_{\xi_{1}=U_{1}\left(x_{1}\right), \ldots, \xi_{d}=U_{d}\left(x_{d}\right)} k_{1}\left(x_{1}\right) \cdots k_{d}\left(x_{d}\right)
$$

is the jump density of a $d$-variate Lévy measure with marginal Lévy densities $k_{1}, \ldots, k_{d}$. 
Using partial integration, we can write the multidimensional Lévy density in terms of the Lévy copula.

Lemma 2.5 Let $f \in C^{d}\left(\mathbb{R}^{d}\right)$ be bounded and vanishing on a neighborhood of the origin. Furthermore, let $X$ be a d-dimensional Lévy process with Lévy measure $v$, Lévy copula $F$, and marginal Lévy measures $v_{i}, i=1, \ldots, d$. Then

$$
\begin{aligned}
\int_{\mathbb{R}^{d}} f(z) v(\mathrm{~d} z)= & \sum_{j=1}^{d} \int_{\mathbb{R}} f\left(0+z_{j}\right) v_{j}\left(\mathrm{~d} z_{j}\right) \\
& +\sum_{j=2}^{d} \sum_{\substack{|I|=j \\
I_{1}<\cdots<I_{j}}} \int_{\mathbb{R}^{j}} \frac{\partial^{j} f}{\partial z^{I}}\left(0+z^{I}\right) F^{I}\left(\left(U_{k}\left(z_{k}\right)\right)_{k \in I}\right) \mathrm{d} z^{I} .
\end{aligned}
$$

Proof We proceed by induction with respect to the dimension $d$. For $d=1$, integration by parts yields

$$
\begin{aligned}
\int_{0}^{\infty} f(z) v(\mathrm{~d} z)= & -\lim _{b \rightarrow \infty} f(b) v(\mathcal{I}(b))+\lim _{a \rightarrow 0+} f(a) v(\mathcal{I}(a)) \\
& +\int_{0}^{\infty} \frac{\partial f}{\partial z}(z) v(\mathcal{I}(z)) \mathrm{d} z, \\
\int_{-\infty}^{0} f(z) v(\mathrm{~d} z)= & \lim _{a \rightarrow 0-} f(a) v(\mathcal{I}(a))-\lim _{b \rightarrow-\infty} f(b) v(\mathcal{I}(b)) \\
& -\int_{-\infty}^{0} \frac{\partial f}{\partial z}(z) v(\mathcal{I}(z)) \mathrm{d} z,
\end{aligned}
$$

and since $f$ is bounded,

$$
\int_{\mathbb{R}} f(z) v(\mathrm{~d} z)=f(0) \lim _{a \rightarrow 0+}(v(\mathcal{I}(a))+v(\mathcal{I}(-a)))+\int_{\mathbb{R}} \frac{\partial f}{\partial z}(z) \operatorname{sgn}(z) v(\mathcal{I}(z)) \mathrm{d} z .
$$

Abusing notation, we write

$$
v(\mathbb{R}):=\lim _{a \rightarrow 0+}(v(\mathcal{I}(a))+v(\mathcal{I}(-a)))
$$

With $f$ vanishing on a neighborhood of 0 we therefore find $f(0) v(\mathbb{R})=0$. For the multidimensional case, we use that by [38, Proposition 11.10] the Lévy measure of $X^{I}$ is given by

$$
v^{I}(B)=v\left(\left\{x \in \mathbb{R}^{d}:\left(x_{i}\right)_{i \in I} \in B \backslash\{0\}\right\}\right), \quad B \in \mathcal{B}\left(\mathbb{R}^{|I|}\right) .
$$

We show by induction with respect to the dimension $d$ that 


$$
\begin{aligned}
\int_{\mathbb{R}^{d}} f(z) v(\mathrm{~d} z)= & f(0, \ldots, 0) v(\mathbb{R}, \ldots, \mathbb{R}) \\
& +\sum_{i=1}^{d} \int_{\mathbb{R}} \frac{\partial f}{\partial z_{i}}\left(0, \ldots, z_{i}, \ldots, 0\right) \operatorname{sgn}\left(z_{i}\right) v_{i}\left(\mathcal{I}\left(z_{i}\right)\right) \mathrm{d} z_{i} \\
& +\sum_{i=2}^{d} \sum_{\substack{|I|=i \\
I_{1}<\cdots<I_{i}}} \int_{\mathbb{R}^{i}} \frac{\partial^{i} f}{\partial z^{I}}\left(0+z^{I}\right) \prod_{j \in I} \operatorname{sgn}\left(z_{j}\right) v^{I}\left(\prod_{j \in I} \mathcal{I}\left(z_{j}\right)\right) \mathrm{d} z^{I}
\end{aligned}
$$

With $f(0, \ldots, 0) v(\mathbb{R}, \ldots, \mathbb{R})=0$, the definition of the tail integrals, and Theorem 2.3, we then have the required result.

For the induction step $d-1 \rightarrow d$, using integration by parts and the induction hypothesis, we obtain

$$
\begin{aligned}
& \int_{\mathbb{R}^{d}} f(z) v(\mathrm{~d} z)=\int_{\mathbb{R}^{d-1}} \int_{\mathbb{R}} f\left(z^{\prime}, z_{d}\right) v\left(\mathrm{~d} z^{\prime}, \mathrm{d} z_{d}\right) \\
& =\int_{\mathbb{R}^{d-1}} f\left(z^{\prime}, 0\right) v\left(\mathrm{~d} z^{\prime}, \mathbb{R}\right) \\
& +\int_{\mathbb{R}^{d-1}} \int_{\mathbb{R}} \frac{\partial f}{\partial z_{d}}\left(z^{\prime}, z_{d}\right) \operatorname{sgn}\left(z_{d}\right) v\left(\mathrm{~d} z^{\prime}, \mathcal{I}\left(z_{d}\right)\right) \mathrm{d} z_{d} \\
& =f(0, \ldots, 0) v(\mathbb{R}, \ldots, \mathbb{R}) \\
& +\sum_{i=1}^{d-1} \int_{\mathbb{R}} \frac{\partial f}{\partial z_{i}}\left(0, \ldots, z_{i}, \ldots, 0\right) \operatorname{sgn}\left(z_{i}\right) v_{i}\left(\mathcal{I}\left(z_{i}\right)\right) \mathrm{d} z_{i} \\
& +\sum_{i=2}^{d-1} \sum_{\substack{|I|=i \\
I_{1}<\cdots<I_{i}}} \int_{\mathbb{R}^{i}} \frac{\partial^{i} f}{\partial z^{I}}\left(0+z^{I}\right) \prod_{j \in I} \operatorname{sgn}\left(z_{j}\right) v^{I}\left(\prod_{j \in I} \mathcal{I}\left(z_{j}\right)\right) \mathrm{d} z^{I} \\
& +\int_{\mathbb{R}} \frac{\partial f}{\partial z_{d}}\left(0, \ldots, 0, z_{d}\right) \operatorname{sgn}\left(z_{d}\right) v\left(\mathbb{R}, \ldots, \mathbb{R}, \mathcal{I}\left(z_{d}\right)\right) \\
& +\sum_{i=1}^{d-1} \int_{\mathbb{R}} \int_{\mathbb{R}} \frac{\partial^{2} f}{\partial z_{i} z_{d}}\left(0, \ldots, z_{i}, \ldots, 0, z_{d}\right) \operatorname{sgn}\left(z_{i}\right) \operatorname{sgn}\left(z_{d}\right) \\
& \times v_{i, d}\left(\mathcal{I}\left(z_{i}\right), \mathcal{I}\left(z_{d}\right)\right) \mathrm{d} z_{i} \mathrm{~d} z_{d} \\
& +\sum_{i=2}^{d-1} \sum_{\substack{|I|=i \\
I_{1}<\cdots<I_{i}}} \int_{\mathbb{R}^{i}} \int_{\mathbb{R}} \frac{\partial^{i+1} f}{\partial z^{I} z_{d}}\left(z^{\{I, d\}}\right) \prod_{j \in\{I, d\}} \operatorname{sgn}\left(z_{j}\right) v^{\{I, d\}} \\
& \times\left(\prod_{j \in\{I, d\}} \mathcal{I}\left(z_{j}\right)\right) \mathrm{d} z^{I} \mathrm{~d} z_{d}
\end{aligned}
$$

which is the claimed result. 
Remark 2.6 The boundedness assumption on $f$ in Lemma 2.5 can be weakened to certain unbounded $f \in C^{d}\left(\mathbb{R}^{d}\right)$ if the Lévy measure $v$ decays sufficiently fast.

Using Lemma 2.5, we immediately obtain the following:

Corollary 2.7 Let $X=\left(X^{1}, \ldots, X^{d}\right)^{\top}$ be a d-dimensional Lévy process with characteristic triplet $(0, v, \gamma)$. Then,

$$
\operatorname{Cov}\left(X^{i}, X^{j}\right)=\int_{\mathbb{R}^{d}} z_{i} z_{j} v(\mathrm{~d} z)=\int_{\mathbb{R}^{2}} F^{\{i, j\}}\left(U_{i}\left(z_{i}\right), U_{j}\left(z_{j}\right)\right) \mathrm{d} z_{i} \mathrm{~d} z_{j}, \quad \forall i \neq j,
$$

where $F$ is the Lévy copula from Theorem 2.3.

We conclude this introductory section with examples of Lévy copulas.

Example 2.8 Examples of Lévy copulas are:

1. Independence Lévy copula,

$$
F\left(u_{1}, \ldots, u_{d}\right)=\sum_{i=1}^{d} u_{i} \prod_{j \neq i} 1_{\{\infty\}}\left(u_{j}\right)
$$

2. Complete dependence Lévy copula,

$$
F\left(u_{1}, \ldots, u_{d}\right)=\min \left(\left|u_{1}\right|, \ldots,\left|u_{d}\right|\right) 1_{K}\left(u_{1}, \ldots, u_{d}\right) \prod_{j=1}^{d} \operatorname{sgn} u_{j},
$$

where $K:=\left\{x \in \mathbb{R}^{d}: \operatorname{sgn}\left(x_{1}\right)=\cdots=\operatorname{sgn}\left(x_{d}\right)\right\}$.

3. Clayton Lévy copulas,

$$
F\left(u_{1}, \ldots, u_{d}\right)=2^{2-d}\left(\sum_{i=1}^{d}\left|u_{i}\right|^{-\theta}\right)^{-\frac{1}{\theta}}\left(\eta 1_{\left\{u_{1} \cdots u_{d} \geq 0\right\}}-(1-\eta) 1_{\left\{u_{1} \cdots u_{d} \leq 0\right\}}\right),
$$

where $\theta>0$ and $\eta \in[0,1]$. For $\eta=1$ and $\theta \rightarrow 0, F$ converges to the independence Lévy copula, for $\eta=1$ and $\theta \rightarrow \infty$, to the complete dependence Lévy copula.

An important class of Lévy copulas are so-called 1-homogeneous copulas.

Definition 2.9 A Lévy copula is called 1-homogeneous if for any $r>0$, there holds

$$
F\left(r u_{1}, \ldots, r u_{d}\right)=r F\left(u_{1}, \ldots, u_{d}\right)
$$

for all $\left(u_{1}, \ldots, u_{d}\right)^{\top} \in \mathbb{R}^{d}$.

For further details and examples of Lévy copulas, we refer to [20, 25]. 


\subsection{Variational parabolic problems}

The bilinear form $\mathcal{E}(\cdot, \cdot)$ associated to $X$ is the basis for the variational formulation of the Kolmogorov equation (1.3), which we now describe. The variational formulation is, in turn, the basis for Galerkin discretizations of the Kolmogorov equations.

To cover equations arising from optimal stopping (as, e.g., for American-style contracts) and from optimal control problems (as, e.g., in portfolio optimization and for options of game type), and in order to accommodate rough payoff functions, the rather general variational framework from $[5,6,21]$ is adopted. The variational setting will be based on the real Gelfand triple with Hilbert space $\mathcal{H}$, i.e.,

$$
\mathcal{V} \subset \mathcal{H} \equiv \mathcal{H}^{*} \subset \mathcal{V}^{*}
$$

For $\mathcal{V}$, we have in mind the domain of $\mathcal{E}(\cdot, \cdot)$. For the infinitesimal generator $\mathcal{A}$ of $X$ and the corresponding bilinear form

$$
\mathcal{E}(u, v):=(\mathcal{A} u, v), \quad u, v \in \mathcal{V},
$$

we assume that there exist constants $C_{1}, C_{2}>0$ and $\lambda \geq 0$ such that for all $u, v \in \mathcal{V}$, there holds

$$
\begin{aligned}
\forall u, v \in \mathcal{V}: & |\mathcal{E}(u, v)| \leq C_{1}\|u\|_{\mathcal{V}}\|v\| \mathcal{V}, \\
\forall u \in \mathcal{V}: & \mathcal{E}(u, u) \geq C_{2}\|u\|_{\mathcal{V}}^{2}-\lambda\|u\|_{\mathcal{H}}^{2} .
\end{aligned}
$$

Moreover, we denote by $(\cdot, \cdot)$ the $\mathcal{H}$ inner product, which admits a unique extension by continuity to $\mathcal{V}^{*} \times \mathcal{V}$ in (2.5). For clarity, we denote this extension by $\langle\cdot, \cdot\rangle^{*} \times \mathcal{V}$.

As already illustrated in the introduction, prices of European-style contracts are solutions of Kolmogorov equations. Their abstract variational formulation reads as follows: Given an initial value $u_{0} \in \mathcal{H}$ and $f \in L^{2}\left((0, T) ; \mathcal{V}^{*}\right)$,

$$
\begin{aligned}
& \text { find } u \in L^{2}((0, T) ; \mathcal{V}) \cap H^{1}\left((0, T) ; \mathcal{V}^{*}\right) \text { such that } \\
& \left\langle\frac{\partial u}{\partial t}, v\right\rangle_{\mathcal{V}^{*} \times \mathcal{V}}+\mathcal{E}(u, v)=\langle f, v\rangle_{\mathcal{V}^{*} \times \mathcal{V}} \quad \forall v \in \mathcal{V}, \text { a.e. in }(0, T), \\
& u(0)=u_{0} \quad \text { in } \mathcal{H}
\end{aligned}
$$

Theorem 2.10 Assume that the bilinear form $\mathcal{E}(\cdot, \cdot)$ satisfies (2.6) and (2.7). Then the abstract parabolic problem (2.8)-(2.9) admits a unique solution.

Proof See, e.g., [27, Theorem 4.1].

Remark 2.11 If instead of a Lévy process $X$, one considers a general strong Markov process with time-dependent infinitesimal generator $\mathcal{A}(t)$ and corresponding bilinear form $\mathcal{E}(t ; u, v)=(\mathcal{A}(t) u, v)$, then Theorem 2.10 remains valid, provided that for all $u, v \in \mathcal{V}$, the mapping $t \mapsto \mathcal{E}(t, u, v)$ is measurable. 
Remark 2.12 The initial condition $u(0)=u_{0}$ is required to hold in $\mathcal{H}$, not in $\mathcal{V}$. Due to the embedding $L^{2}((0, T) ; \mathcal{V}) \cap H^{1}\left((0, T) ; \mathcal{V}^{*}\right) \subset C^{0}([0, T] ; \mathcal{H})$, the initial condition (2.9) makes sense, and the parabolic evolution problem is well posed even for initial data $u_{0}$ belonging to $\mathcal{H}$ but not to $\mathcal{V}$. For example, this is the case in European derivative contracts with discontinuous payoffs, such as binary options.

For the study of optimal stopping problems which arise, e.g., from American contracts, we require variational formulations of parabolic variational inequalities. To this end, let $\emptyset \neq \mathcal{K} \subset \mathcal{V}$ be a closed, nonempty, and convex subset of $\mathcal{V}$ with the indicator function

$$
\phi(v):=I_{\mathcal{K}}(v)= \begin{cases}0, & \text { if } v \in \mathcal{K} \\ +\infty, & \text { else. }\end{cases}
$$

This is a proper, convex, lower semicontinuous (1.s.c.) function $\phi: \mathcal{V} \rightarrow \overline{\mathbb{R}}$ with domain $\mathcal{D}(\phi)=\{v \in \mathcal{V}: \phi(v)<\infty\}$. We denote by $\overline{\mathcal{K}}^{\|\circ\|_{\mathcal{H}}}$ the closure of $\mathcal{D}(\phi)$ in $\mathcal{H}$ and consider the following variational problem: Given $f \in L^{2}\left((0, T) ; \mathcal{V}^{*}\right)$, $u_{0} \in \overline{\mathcal{K}}^{\|\circ\|_{\mathcal{H}}} \subset \mathcal{H}$

find $u \in L^{2}((0, T) ; \mathcal{V}) \cap H^{1}\left((0, T) ; \mathcal{V}^{*}\right)$ such that $u \in \mathcal{D}(\phi)$ a.e. in $(0, T)$ and

$$
\begin{aligned}
& \left\langle\frac{\partial u}{\partial t}+\mathcal{A} u-f, u-v\right\rangle_{\mathcal{V}^{*} \times \mathcal{V}}+\phi(u)-\phi(v) \geq 0 \quad \forall v \in \mathcal{D}(\phi) \text { a.e. in }(0, T), \\
& u(0)=u_{0} \quad \text { in } \mathcal{H} .
\end{aligned}
$$

Existence and uniqueness results for solutions $u \in L^{2}((0, T) ; \mathcal{V})$ of $(2.11)-(2.12)$ can be obtained from, e.g., [21, Theorem 6.2.1] under rather strict conditions on the data $f(t)$. To derive the well-posedness of (2.11)-(2.12) under minimal regularity conditions on $f(t), u_{0}$, and $\phi$, the problem needs to be replaced by a weak variational formulation. To state it, introduce the integral functional $\Phi$ on $L^{2}((0, T) ; \mathcal{V})$ given by

$$
\Phi(v)= \begin{cases}\int_{0}^{T} \phi(v(t)) e^{-2 \lambda t} \mathrm{~d} t, & \text { if } \phi(v) \in L^{1}(0, T), \\ +\infty, & \text { else, }\end{cases}
$$

with $\lambda \geq 0$ as in (2.7). Note that $\Phi(\cdot)$ is proper, convex, and l.s.c. with domain

$$
\mathcal{D}(\Phi)=\left\{v \in L^{2}((0, T) ; \mathcal{V}): \phi(v) \in L^{1}(0, T)\right\}
$$

Then the weak variational formulation of (2.11)-(2.12) reads (cf. [2, 39]) as follows: 


$$
\text { Given } u_{0} \in \overline{\mathcal{K}}^{\|\circ\|_{\mathcal{H}}} \subset \mathcal{H} \quad \text { and } \quad f \in L^{2}\left((0, T) ; \mathcal{V}^{*}\right) \text {, }
$$

find $u \in L^{\infty}((0, T) ; \mathcal{H}) \cap \mathcal{D}(\Phi)$ such that $u(0)=u_{0}$ in $\mathcal{H} \quad$ and

$$
\begin{aligned}
& \int_{0}^{T}\left\langle\frac{\partial v}{\partial t}(t)+(\mathcal{A}+\lambda) u(t)-(f(t)+\lambda v(t)), u(t)-v(t)\right\rangle e^{-2 \lambda t} \mathrm{~d} t+\Phi(u)-\Phi(v) \\
& \quad \leq \frac{1}{2}\left\|u_{0}-v(0)\right\|_{\mathcal{H}}^{2}
\end{aligned}
$$

for all $v \in \mathcal{D}(\Phi)$ with $\frac{\partial v}{\partial t} \in L^{2}\left((0, T) ; \mathcal{V}^{*}\right)$.

The well-posedness of (2.13) is ensured by [39, Theorem 4.1]:

Theorem 2.13 Assume that the bilinear form $\mathcal{E}(\cdot, \cdot)$ satisfies (2.6)-(2.7). Then the problem (2.13) admits a unique solution

$$
u \in L^{2}((0, T) ; \mathcal{V}) \cap L^{\infty}((0, T) ; \mathcal{H}) \text { such that } t \mapsto \phi(u(t, \cdot)) \in L^{1}(0, T) .
$$

Remark 2.14 As for the parabolic equality problem (2.8)-(2.9), also for (2.13), the initial condition is only required to hold in $\mathcal{H}$. In addition, however, in (2.13) the data $u_{0}$ must belong to the closure $\overline{\mathcal{K}}^{\left\|^{\circ}\right\|_{\mathcal{H}}}$ of $\mathcal{K}$ in $\mathcal{H}$.

Remark 2.15 Convergence rates for backward Euler time discretizations of the weak variational problem (2.13) for American-style contracts under minimal regularity are given in $[2,31,39]$.

\section{Properties of Lévy measures built from Lévy copulas}

In the present section, we verify properties of Lévy measures corresponding to multivariate Lévy processes $X$ with state space $\mathbb{R}^{d}$ built from so-called tempered stable, univariate Lévy processes $X^{i}$ by 1 -homogeneous Lévy copulas as constructed in Sect. 2.2. For the (in general nonsymmetric) bilinear form $\mathcal{E}(\cdot, \cdot)$ corresponding to the generator $\mathcal{A}$ of $X$, we verify the so-called sector condition. Due to a classical result of Berg and Forst [3] (see also [22, Chap. 4.7]) this, in conjunction with the translation invariance of $X$, implies that $\mathcal{E}(\cdot, \cdot)$ is a nonsymmetric Dirichlet form. It also allows us to give an explicit characterization of the domains $\mathcal{D}(\mathcal{A})$ and $\mathcal{D}(\mathcal{E})$ of $\mathcal{A}$ and $\mathcal{E}(\cdot, \cdot)$ in terms of anisotropic Sobolev spaces.

\subsection{Semiheavy tails}

At first, we show that the tails of the multivariate Lévy processes stemming from the copula construction decay exponentially fast, provided that the one-dimensional marginal processes are of tempered stable type in the sense of [4], i.e., the corresponding densities decay exponentially at infinity.

We use the following assumptions on the marginal Lévy measures $v_{i}, i=1, \ldots, d$. These are satisfied by a wide range of Lévy models [29]. 
Assumption 3.1 Let $X$ be a Lévy process with state space $\mathbb{R}^{d}$, characteristic triplet $(\mathcal{Q}, v, \gamma)$, and marginal Lévy measures $v_{i}, i=1, \ldots, d$, with densities $k_{i}$. There are constants $G_{i}>0, M_{i}>0, i=1, \ldots, d$ such that

$$
k_{i}(z) \lesssim \begin{cases}e^{-G_{i}|z|}, & z<-1, \\ e^{-M_{i} z}, & z>1\end{cases}
$$

The tail behavior (3.1) carries over to the $d$-variate case.

Proposition 3.2 Let $X$ be a Lévy process with state space $\mathbb{R}^{d}$ and Lévy measure $v$ such that the marginal measures $v_{i}$ satisfy (3.1). Then the Lévy measure $v$ also decays exponentially, i.e.,

$$
\int_{|z|>1} e^{\eta(z)} v(\mathrm{~d} z)<\infty, \quad \text { with } \eta(z)=\sum_{i=1}^{d}\left(\mu_{i}^{+} 1_{\left\{z_{i}>0\right\}}+\mu_{i}^{-} 1_{\left\{z_{i}<0\right\}}\right)\left|z_{i}\right|,
$$

where $0<\mu_{i}^{-}<\frac{G_{i}}{d}, 0<\mu_{i}^{+}<\frac{M_{i}}{d}, i=1, \ldots, d$. For each $i=1, \ldots, d$, there holds

$$
\int_{|z|>1} e^{\eta_{i}(z)} v(\mathrm{~d} z)<\infty, \quad \text { with } \eta_{i}(z)=\left(\mu_{i}^{+} 1_{\left\{z_{i}>0\right\}}+\mu_{i}^{-} 1_{\left\{z_{i}<0\right\}}\right)\left|z_{i}\right|,
$$

where now $0<\mu_{i}^{-}<G_{i}$ and $0<\mu_{i}^{+}<M_{i}, i=1, \ldots, d$. Furthermore, the density $p_{t}(x)$ of the process $X$ at time $t>0$ also decays exponentially, independently of $t$, i.e.,

$$
\int_{\mathbb{R}^{d}} e^{\eta_{i}(x)} p_{t}(x) \mathrm{d} x<\infty, \quad \text { with } \eta_{i}(z)=\left(\mu_{i}^{+} 1_{\left\{z_{i}>0\right\}}+\mu_{i}^{-} 1_{\left\{z_{i}<0\right\}}\right)\left|z_{i}\right|,
$$

where $0<\mu_{i}^{-}<G_{i}$ and $0<\mu_{i}^{+}<M_{i}, i=1, \ldots, d$.

Proof Using [38, Proposition 11.10] as in Lemma 2.5, we obtain

$$
\begin{aligned}
\int_{|z|>1} e^{\sum_{i=1}^{d} \mu_{i}\left|z_{i}\right|} v(\mathrm{~d} z) & \lesssim \sum_{i=1}^{d} \int_{|z|>1} e^{d \mu_{i}\left|z_{i}\right|} v(\mathrm{~d} z) \\
& \lesssim \sum_{i=1}^{d} \int_{\left|z_{i}\right|>1} e^{d \mu_{i}\left|z_{i}\right|} v_{i}\left(\mathrm{~d} z_{i}\right)<\infty
\end{aligned}
$$

where $\mu_{i}$ can be chosen as $\mu_{i}^{-}$or $\mu_{i}^{+}, i=1, \ldots, d$. Equation (3.2) follows from Sato [38, Theorem 25.3].

\subsection{Sector condition}

We verify here for the characteristic exponents of the Lévy processes the so-called sector condition, i.e.,

$$
\exists C>0:|\Im \psi(\xi)| \leq C \Re \psi(\xi) \text { for all } \xi \in \mathbb{R}^{d} .
$$


Since the bilinear form of the Lévy process is in general a nonsymmetric bilinear form due to the asymmetric jump structure in financial models, this condition is necessary for the bilinear form to be a Dirichlet form. Additionally, it allows us to give an explicit characterization of the domains $\mathcal{D}(\mathcal{A})$ and $\mathcal{D}(\mathcal{E})$ of the infinitesimal generator and bilinear form of $X$, cf. [3] and [22, Example 4.7.32].

Assumption 3.3 Let $X$ be a Lévy process with state space $\mathbb{R}^{d}$, characteristic triplet $(\mathcal{Q}, v, \gamma)$, and marginal Lévy measures $v_{i}, i=1, \ldots, d$ with densities $k_{i}$. There are constants $0<Y_{i}<2$ and $c_{i}^{+}, c_{i}^{-} \geq 0, c_{i}^{+}+c_{i}^{-}>0, i=1, \ldots, d$, such that

$$
\begin{array}{ll}
k_{i}(z) \gtrsim c_{i}^{-} \frac{1}{|z|^{1+Y_{i}}} 1_{\{z<0\}}(z)+c_{i}^{+} \frac{1}{z^{1+Y_{i}}} 1_{\{0<z\}}(z), \quad 0<|z| \leq 1, \\
k_{i}(z) \lesssim c_{i}^{-} \frac{1}{|z|^{1+Y_{i}}} 1_{\{z<0\}}(z)+c_{i}^{+} \frac{1}{z^{1+Y_{i}}} 1_{\{0<z\}}(z), \quad 0<|z| \leq 1 .
\end{array}
$$

Example 3.4 Assumption 3.3 coincides with assumptions (A1), (A4) in [29, Sect. 3.2]. It is shown that these are satisfied by a wide range of processes, including the generalized hyperbolic, Meixner, and tempered stable processes. Here, we just mention the nonsymmetric tempered stable (CGMY) processes as in [9] and spectrally negative processes where the marginal densities are given by

$$
k_{i}(z)=\left\{\begin{array}{ll}
C_{i} \frac{e^{-G_{i}|z|}}{|z|^{1+Y_{i}}}, & z<0, \\
C_{i} \frac{e^{-M_{i} z}}{z^{1+Y_{i}}}, & z>0,
\end{array} \quad \text { and } \quad k_{i}(z)= \begin{cases}C_{i} \frac{e^{-G_{i}|z|}}{|z|^{1+Y_{i}}}, & z<0 \\
0, & z>0\end{cases}\right.
$$

with $G_{i}, M_{i} \geq 0, i=1, \ldots, d$. An overview over different Lévy densities can be found in [41].

The following proposition provides an upper bound for $|\psi(\xi)|$ and hence for $|\Im \psi(\xi)|$.

Proposition 3.5 Let $X$ be a Lévy process with state space $\mathbb{R}^{d}$, characteristic triplet $(\mathcal{Q}, v, \gamma)$, and characteristic exponent $\psi$. Assume that $\mathcal{Q}=0$ and $\gamma_{i}=0$, $i=1, \ldots, d$, and that the marginal Lévy measures $v_{i}, i=1, \ldots, d$, satisfy (3.5). Then for $\|\xi\|_{\infty}>1$, there holds

$$
|\psi(\xi)| \lesssim \sum_{j=1}^{d}\left|\xi_{j}\right|^{Y_{j}}
$$

Proof For notational convenience, we assume without loss of generality that there are only positive jumps. We distinguish the cases of $Y_{i}$ smaller or larger than 1 . After possibly renumbering coordinates, let $0 \leq j \leq d$ be such that

$$
Y_{1}, \ldots, Y_{j}<1, \quad 1 \leq Y_{j+1}, \ldots, Y_{d}<2 .
$$


Then the characteristic exponent $\psi$ can be written as

$$
\psi(\xi)=\int_{\mathbb{R}_{\geq 0}^{d}}\left(1-e^{i\langle\xi, z\rangle}+\sum_{k=j+1}^{d} i \xi_{k} z_{k} 1_{|z| \leq 1}\right) v(\mathrm{~d} z)+i \sum_{k=1}^{j} \tilde{\gamma}_{k} \xi_{k} .
$$

Without loss of generality, we set $\tilde{\gamma}_{k}, k=1, \ldots, j$, to zero. With the notation $B=\left[0, \frac{1}{d\left|\xi_{1}\right|}\right] \times \cdots \times\left[0, \frac{1}{d\left|\xi_{d}\right|}\right]$ we obtain

$$
\begin{aligned}
|\psi(\xi)| \lesssim & \int_{[0,1]^{d}}\left|1-e^{i\langle\xi, z\rangle}+\sum_{k=j+1}^{d} i \xi_{k} z_{k}\right| v(\mathrm{~d} z)+1 \\
& \lesssim \int_{B}\left|1-e^{i\langle\xi, z\rangle}+\sum_{k=j+1}^{d} i \xi_{k} z_{k}\right| v(\mathrm{~d} z) \\
& +\int_{[0,1]^{d} \backslash B}\left(1+\sum_{k=j+1}^{d}\left|\xi_{k} z_{k}\right|\right) \nu(\mathrm{d} z)+1 .
\end{aligned}
$$

We estimate the first term via

$$
\begin{aligned}
\int_{B} \mid & 1-e^{i\langle\xi, z\rangle}+\sum_{k=j+1}^{d} i \xi_{k} z_{k} \mid v(\mathrm{~d} z) \\
& \lesssim \int_{B}\left(\sum_{k=1}^{j}\left|\xi_{k} z_{k}\right|+\sum_{k=j+1}^{d} \xi_{k}^{2} z_{k}^{2}\right) v(\mathrm{~d} z) \\
& \lesssim \sum_{k=1}^{j} \int_{0}^{\frac{1}{\left|\xi_{k}\right|}}\left|\xi_{k} z_{k}\right| v_{k}\left(\mathrm{~d} z_{k}\right)+\sum_{k=j+1}^{d} \int_{0}^{\frac{1}{\xi_{k} \mid}} \xi_{k}^{2} z_{k}^{2} v_{k}\left(\mathrm{~d} z_{k}\right) \\
& \lesssim \sum_{k=1}^{j} \int_{0}^{\frac{1}{\left|\xi_{k}\right|}}\left|\xi_{k} z_{k}\right| \frac{1}{z_{k}^{Y_{k}+1}} \mathrm{~d} z_{k}+\sum_{k=j+1}^{d} \int_{0}^{\frac{1}{\xi_{k} \mid}} \xi_{k}^{2} z_{k}^{2} \frac{1}{z_{k}^{Y_{k}+1}} \mathrm{~d} z_{k} \\
& \lesssim \sum_{k=1}^{d}\left|\xi_{k}\right|^{Y_{k}} .
\end{aligned}
$$

To estimate the second term, note that if $z \in[0,1]^{d} \backslash B$ with $z_{k} \leq \frac{1}{d\left|\xi_{k}\right|}$, there exists $\ell_{k}$ such that $z_{\ell_{k}} \geq \frac{1}{d\left|\xi_{\ell_{k}}\right|}$. Hence,

$$
\int_{[0,1]^{d} \backslash B}\left(1+\sum_{k=j+1}^{d}\left|\xi_{k} z_{k}\right|\right) v(\mathrm{~d} z)
$$




$$
\begin{aligned}
\leq & \sum_{k=j+1}^{d} \int_{-\infty}^{\infty} \cdots \int_{\frac{1}{d\left|\xi_{k}\right|}}^{1} \cdots \int_{-\infty}^{\infty}\left(1+\left|\xi_{k} z_{k}\right|\right) v(\mathrm{~d} z) \\
& +\sum_{k=j+1}^{d} \int_{-\infty}^{\infty} \cdots \int_{0}^{\frac{1}{d\left|\xi_{k}\right|}} \cdots \int_{\frac{1}{d\left|\xi_{\ell_{k}}\right|}}^{1} \cdots \int_{-\infty}^{\infty}\left(1+\left|\xi_{k} z_{k}\right|\right) v(\mathrm{~d} z) \\
\leq & \sum_{k=j+1}^{d} \int_{\frac{1}{d\left|\xi_{k}\right|}}^{1}\left(1+\left|\xi_{k} z_{k}\right|\right) v_{k}\left(\mathrm{~d} z_{k}\right) \\
& +\sum_{k=j+1}^{d} \int_{-\infty}^{\infty} \cdots \int_{0}^{\frac{1}{d\left|\xi_{k}\right|}} \cdots \int_{\frac{1}{d \mid \xi_{\ell_{k}}}}^{1} \cdots \int_{-\infty}^{\infty}\left(1+\frac{1}{d}\right) v(\mathrm{~d} z) \\
\lesssim & +\sum_{k=j+1}^{d}\left|\xi_{k}\right|^{Y_{k}}+\sum_{k=j+1}^{d}\left|\xi_{k}\right|+\sum_{k=j+1}^{1} \int_{\frac{1}{d\left|\xi_{k}\right|}}^{1} v_{\ell_{k}}\left(\mathrm{~d} z_{\ell_{k}}\right) \\
\lesssim & +\sum_{k=1}^{d}\left|\xi_{k}\right|^{Y_{k}}+\sum_{k=j+1}^{d}\left|\xi_{k}\right| .
\end{aligned}
$$

Therefore, we obtain, for $\|\xi\|_{\infty}>1$,

$$
|\psi(\xi)| \lesssim \sum_{k=1}^{d}\left|\xi_{k}\right|^{Y_{k}}
$$

In order to prove (3.3), we also require a lower bound on $\Re \psi(\xi)$. For this, we need to make a few technical assumptions on the underlying copula $F$. To state these assumptions, we introduce some notation.

Definition 3.6 Let $\mathcal{I} \subset \mathbb{R}$. Two functions $f, g: \mathbb{R} \rightarrow \mathbb{R}$ are called equivalent on $\mathcal{I}$ if there exists a constant $c>0$ such that

$$
c|f(x)| \leq|g(x)| \leq c^{-1}|f(x)| \text { for all } x \in \mathcal{I} \text {. }
$$

We denote the equivalence of $f$ and $g$ by $f \sim g$.

Definition 3.7 A function $F: \overline{\mathbb{R}}^{d} \rightarrow \overline{\mathbb{R}}$ is called equivalence preserving if, for any two families of equivalent functions $f_{i} \sim g_{i}, i=1, \ldots, d$, on some $\mathcal{I} \subset \mathbb{R}$, there exists a constant $C>0$ such that

$$
C F\left(f_{1}\left(x_{1}\right), \ldots, f_{d}\left(x_{d}\right)\right) \leq F\left(g_{1}\left(x_{1}\right), \ldots, g_{d}\left(x_{d}\right)\right) \leq C^{-1} F\left(f_{1}\left(x_{1}\right), \ldots, f_{d}\left(x_{d}\right)\right)
$$

for all $x \in \mathcal{I}^{d}$.

We can now state the sufficient assumptions on the Lévy copula. 
Assumption 3.8 Let $X$ be a Lévy process with state space $\mathbb{R}^{d}$. Assume that the Lévy copula $F$ is 1 -homogeneous and that the derivative $\partial_{1} \cdots \partial_{d} F: \overline{\mathbb{R}}^{d} \rightarrow \overline{\mathbb{R}}$ exists in the sense of distributions (i.e., the multivariate process admits a Lévy kernel) and is equivalence preserving.

One readily infers that, for instance, the independence copula (2.4) satisfies Assumption 3.8. Nonetheless, the equivalence-preserving property of $\partial_{1} \cdots \partial_{d} F$ is nontrivial in general. We prove it for a wide class of Lévy copulas in Appendix, but first, under Assumption 3.8, one obtains the required lower bound of $\Re \psi(\xi)$ :

Proposition 3.9 Let $X$ be a Lévy process with state space $\mathbb{R}^{d}$, characteristic triplet $(\mathcal{Q}, v, \gamma)$, and characteristic exponent $\psi$. Assume that $\mathcal{Q}=0$ and that the marginal Lévy measures $v_{i}, i=1, \ldots, d$, satisfy (3.4), and the Lévy copula $F$ satisfies Assumption 3.8. Then, for $\|\xi\|_{\infty}$ sufficiently large,

$$
\Re \psi(\xi) \gtrsim \sum_{j=1}^{d}\left|\xi_{j}\right|^{Y_{j}}
$$

Proof First, let $d=1$. Using $1-\cos (z)=2\left(\sin \frac{z}{2}\right)^{2} \gtrsim z^{2}$ for $|z| \leq 1$, we obtain for $|\xi|>1$ that

$$
\Re \psi(\xi)=\int_{\mathbb{R}}(1-\cos (\xi z)) k(z)(\mathrm{d} z) \gtrsim \int_{-\frac{1}{|\xi|}}^{\frac{1}{|\xi|}} \xi^{2} z^{2} k(z) \mathrm{d} z \gtrsim|\xi|^{Y} .
$$

Now let $d>1$ and suppose that Assumption 3.8 is satisfied. Consider the kernels

$$
k_{i}^{0}(z):=c_{i}^{-} \frac{1}{z^{1+Y_{i}}} 1_{\{z<0\}}(z)+c_{i}^{+} \frac{1}{z^{1+Y_{i}}} 1_{\{0 \leq z\}}(z), \quad i=1, \ldots, d,
$$

where $Y_{i}, c_{i}^{+}, c_{i}^{-}$are the constants of (3.4). Denote by $U_{i}: \mathbb{R} \rightarrow \mathbb{R}$ the marginal tail integrals of $X$ and let $U_{i}^{0}$ be the tail integral corresponding to $k_{i}^{0}$. From (3.4)-(3.5) one infers $k_{i} \sim k_{i}^{0}$ and $U_{i} \sim U_{i}^{0}$ on $[-1,1], i=1, \ldots, d$. By Assumption 3.8, Remark 2.4 yields that the Lévy measure $v$ of $X$ admits a kernel representation $v(\mathrm{~d} x)=k(x) \mathrm{d} x$ with

$$
k\left(x_{1}, \ldots, x_{d}\right)=\left(\partial_{1} \cdots \partial_{d} F\right)(\underline{U}(x)) k_{1}\left(x_{1}\right) \cdots k_{d}\left(x_{d}\right),
$$

where we have set $\underline{U}(x)=\left(U_{1}\left(x_{1}\right), \ldots, U_{d}\left(x_{d}\right)\right)$. Thus, using the equivalencepreserving property of $\partial_{1} \cdots \partial_{d} F$, one obtains

$$
\begin{aligned}
\Re \psi(\xi) & =\int_{\mathbb{R}^{d}}(1-\cos \langle\xi, x\rangle) k(x) \mathrm{d} x \\
& \geq \int_{B_{1}(0)}(1-\cos \langle\xi, x\rangle)\left(\partial_{1} \cdots \partial_{d} F\right)(\underline{U}(x)) k_{1}\left(x_{1}\right) \cdots k_{d}\left(x_{d}\right) \mathrm{d} x \\
& \geq C \int_{B_{1}(0)}(1-\cos \langle\xi, x\rangle)\left(\partial_{1} \cdots \partial_{d} F\right)\left(\underline{U^{0}}(x)\right) k_{1}^{0}\left(x_{1}\right) \cdots k_{d}^{0}\left(x_{d}\right) \mathrm{d} x .
\end{aligned}
$$


Now define $k^{0}\left(x_{1}, \ldots, x_{d}\right):=\left(\partial_{1} \cdots \partial_{d} F\right)\left(\underline{U^{0}}(x)\right) k_{1}^{0}\left(x_{1}\right) \ldots k_{d}^{0}\left(x_{d}\right)$. Since $F$ is 1 -homogeneous and the marginal kernels $k_{i}^{0}$ satisfy the homogeneity condition

$$
k_{i}^{0}(r z)=r^{-1-Y_{i}} k_{i}^{0}(z) \quad \text { for all } r>0, z \in \mathbb{R} \backslash\{0\},
$$

by [20, Theorem 3.2] there holds

$$
k^{0}\left(r^{-\frac{1}{Y_{1}}} x_{1}, \ldots, r^{-\frac{1}{Y_{d}}} x_{d}\right)=r^{1+\frac{1}{Y_{1}}+\cdots+\frac{1}{Y_{d}}} k^{0}\left(x_{1}, \ldots, x_{d}\right)
$$

for all $r>0$ and $x \in \mathbb{R}^{d}$ such that $x_{i} \neq 0$. Using [20, Theorem 3.3], one obtains that $\psi^{0}(\xi):=\int_{\mathbb{R}^{d}}(1-\cos \langle\xi, z\rangle) k^{0}(x) \mathrm{d} x$ is an anisotropic distance function of or$\operatorname{der}\left(1 / Y_{1}, \ldots, 1 / Y_{d}\right)$. Since all anisotropic distance functions of the same order are equivalent (cf., e.g., [16, Lemma 2.2]), there exists some constant $C_{1}>0$ such that

$$
\psi^{0}(\xi) \geq C_{1}\left(\left|\xi_{1}\right|^{Y_{1}}+\cdots+\left|\xi_{d}\right|^{Y_{d}}\right) \quad \text { for all } \xi \in \mathbb{R}^{d} .
$$

Hence, by (3.6),

$$
\begin{aligned}
\Re \psi(\xi) & \geq C \psi^{0}(\xi)-C \int_{\mathbb{R}^{d} \backslash B_{1}(0)}(1-\cos \langle\xi, x\rangle) k^{0}(x) \mathrm{d} x \\
& \geq C \psi^{0}(\xi)-2 C \int_{\mathbb{R}^{d} \backslash B_{1}(0)} k^{0}(x) \mathrm{d} x \\
& \geq C \psi^{0}(\xi)-C^{\prime} \\
& \geq C C_{1} \sum_{i=1}^{d}\left|\xi_{i}\right|^{Y_{i}}-C^{\prime} .
\end{aligned}
$$

Since $\psi$ is continuous, we immediately obtain the sector condition.

Theorem 3.10 Let $X$ be a Lévy process with state space $\mathbb{R}^{d}$, characteristic triplet $(\mathcal{Q}, v, \gamma)$, and characteristic exponent $\psi$. Assume that either $\mathcal{Q}>0$ or $\mathcal{Q}=0$ and $\gamma_{i}=0, i=1, \ldots, d$, and in the latter case that the marginal Lévy measures $v_{i}, i=1, \ldots, d$, satisfy (3.4)-(3.5) and the Lévy copula F satisfies Assumption 3.8. Then

$$
|\Im \psi(\xi)| \lesssim \Re \psi(\xi) \quad \forall \xi \in \mathbb{R}^{d}
$$

Proof For $\mathcal{Q}=0$, the result follows with Propositions 3.5 and 3.9. For $\mathcal{Q}>0$, we have

$$
\begin{aligned}
\Re \psi(\xi) & =\frac{1}{2}\langle\xi, \mathcal{Q} \xi\rangle+\int_{\mathbb{R}^{d}}(1-\cos \langle\xi, z\rangle) v(\mathrm{~d} z) \\
& \gtrsim \sum_{j=1}^{d} \xi_{j}^{2},
\end{aligned}
$$


and for $\|\xi\|_{\infty}>1$,

$$
|\psi(\xi)| \lesssim|\langle\gamma, \xi\rangle|+\langle\xi, \mathcal{Q} \xi\rangle+\int_{\mathbb{R}^{d}}\left|e^{i\langle\xi, z\rangle}-1-i\langle\xi, z\rangle 1_{|z| \leq 1}\right| v(\mathrm{~d} z) \lesssim \sum_{j=1}^{d} \xi_{j}^{2}
$$

Thus, the result follows from the continuity of $\psi$.

\section{Option pricing}

Assume that the risk-neutral dynamics of $d \geq 1$ assets are given by

$$
S_{t}^{i}=S_{0}^{i} e^{r t+X_{t}^{i}}, \quad i=1, \ldots, d,
$$

where $X$ is a $d$-variate Lévy process with characteristic triplet $\left(\mathcal{Q}, v_{\mathbb{Q}}, \gamma\right)$ under a risk-neutral measure $\mathbb{Q}$ such that $e^{X^{i}}$ is a martingale with respect to the canonical filtration $\mathcal{F}_{t}^{0}:=\sigma\left(X_{s}, s \leq t\right), t \geq 0$, of the multivariate process $X$. As shown in Lemma 2.1, this martingale condition implies

$$
\int_{|z|>1} e^{z_{i}} \nu_{\mathbb{Q}}(\mathrm{d} z)<\infty, \quad i=1, \ldots, d .
$$

This property holds for semiheavy tails satisfying (3.1) with $M_{i}>1, i=1, \ldots, d$, as shown in Proposition 3.2. We drop the subscript $\mathbb{Q}$ in what follows.

Remark 4.1 Note that $e^{X^{i}}$ is also a martingale with respect to the filtration $\sigma\left(X_{s}^{i}, s \leq t\right)$ associated to the $i$ th marginal process $X^{i}$.

\subsection{Partial integro-differential equations (PIDEs) for European contracts}

We consider a European option with maturity $T<\infty$ and payoff $g\left(S_{T}\right)$, which is assumed to be Lipschitz. The value $V(t, s)$ of this option is given by

$$
V(t, s)=\mathbb{E}\left(e^{-r(T-t)} g\left(S_{T}\right) \mid S_{t}=s\right) .
$$

It can be characterized as a solution of a PIDE.

Theorem 4.2 Let $X$ be a Lévy process with state space $\mathbb{R}^{d}$ and characteristic triplet $(\mathcal{Q}, v, \gamma)$. Assume that the function $V(t, s)$ in (4.1) satisfies

$$
V(t, s) \in C^{1,2}\left((0, T) \times \mathbb{R}_{>0}^{d}\right) \cap C^{0}\left([0, T] \times \mathbb{R}_{\geq 0}^{d}\right) .
$$

Then $V(t, s)$ is a classical solution of the backward Kolmogorov equation

$$
\begin{aligned}
& \frac{\partial V}{\partial t}(t, s)+\frac{1}{2} \sum_{i, j=1}^{d} s_{i} s_{j} \mathcal{Q}_{i j} \frac{\partial^{2} V}{\partial s_{i} \partial s_{j}}+r \sum_{i=1}^{d} s_{i} \frac{\partial V}{\partial s_{i}}(t, s)-r V(t, s) \\
& \quad+\int_{\mathbb{R}^{d}}\left(V\left(t, s e^{z}\right)-V(t, s)-\sum_{i=1}^{d} s_{i}\left(e^{z_{i}}-1\right) \frac{\partial V}{\partial s_{i}}(t, s)\right) v(\mathrm{~d} z)=0
\end{aligned}
$$


on $(0, T) \times \mathbb{R}_{>0}^{d}$, where $V\left(t, s e^{z}\right):=V\left(t, s_{1} e^{z_{1}}, \ldots, s_{d} e^{z_{d}}\right)$, and the terminal condition is given by

$$
V(T, s)=g(s) \quad \forall s \in \mathbb{R}_{\geq 0}^{d}
$$

Proof We first need the risk-neutral dynamics of $S^{i}$. Let $\Sigma=\left(\Sigma_{i j}\right)_{1 \leq i, j \leq d}$ be given such that $\Sigma \Sigma^{\top}=\mathcal{Q}$. With the Itô formula, for multidimensional Lévy processes and the Lévy-Itô decomposition, we obtain

$$
\begin{aligned}
& \mathrm{d} S_{t}^{i}=r S_{t}^{i} \mathrm{~d} t+S_{t-}^{i} \mathrm{~d} X_{t}^{i}+\frac{1}{2} \mathcal{Q}_{i i} S_{t}^{i} \mathrm{~d} t+S_{t-}^{i} e^{\Delta X_{t}^{i}}-S_{t-}^{i}-\Delta X_{t}^{i} S_{t-}^{i} \\
& =r S_{t}^{i} \mathrm{~d} t+S_{t-}^{i} \gamma_{i} \mathrm{~d} t+S_{t-}^{i} \sum_{k=1}^{d} \Sigma_{i k} \mathrm{~d} W_{t}^{k}+\int_{|z|<1} S_{t-z_{i}}^{i} \tilde{J}(\mathrm{~d} t, \mathrm{~d} z)+\frac{1}{2} \mathcal{Q}_{i i} S_{t}^{i} \mathrm{~d} t \\
& +S_{t-}^{i}(e^{\Delta X_{t}^{i}}-1 \underbrace{-1}_{-\Delta X_{t}^{i} 1_{\left\{\left|\Delta X_{t}\right|<1\right\}}-\Delta X_{t}^{i}+\Delta X_{t}^{i} 1_{\left\{\left|\Delta X_{t}\right| \geq 1\right\}}}) \\
& =r S_{t}^{i} \mathrm{~d} t+S_{t-}^{i} \gamma_{i} \mathrm{~d} t+S_{t-}^{i} \sum_{k=1}^{d} \Sigma_{i k} \mathrm{~d} W_{t}^{k}+\frac{1}{2} \mathcal{Q}_{i i} S_{t}^{i} \mathrm{~d} t \\
& +\int_{\mathbb{R}^{d}} S_{t-}^{i}\left(e^{z_{i}}-1\right) \tilde{J}(\mathrm{~d} t, \mathrm{~d} z)+\int_{\mathbb{R}^{d}} S_{t-}^{i}\left(e^{z_{i}}-1-z_{i} 1_{\{|z|<1\}}\right) v(\mathrm{~d} z) \mathrm{d} t .
\end{aligned}
$$

Since $e^{X^{i}}$ is a martingale, we have

$$
\mathrm{d} S_{t}^{i}=r S_{t}^{i} \mathrm{~d} t+S_{t-}^{i} \sum_{k=1}^{d} \Sigma_{i k} \mathrm{~d} W_{t}^{k}+\int_{\mathbb{R}^{d}} S_{t-}^{i}\left(e^{z_{i}}-1\right) \tilde{J}(\mathrm{~d} t, \mathrm{~d} z)
$$

We now apply the Itô formula for semimartingales [24, Theorem 4.57] to the discounted values $e^{-r t} V_{t}$. This gives

$$
\begin{aligned}
\mathrm{d}\left(e^{-r t} V_{t}\right)= & -r e^{-r t} V \mathrm{~d} t+e^{-r t}\left(\frac{\partial V}{\partial t}\left(t, S_{t}\right) \mathrm{d} t+\sum_{i=1}^{d} \frac{\partial V}{\partial s_{i}}\left(t, S_{t-}\right) \mathrm{d} S_{t}^{i}\right. \\
& +\frac{1}{2} \sum_{i, j=1}^{d} \frac{\partial^{2} V}{\partial s_{i} \partial s_{j}}\left(t, S_{t-}\right) \mathrm{d}\left[S^{i}, S^{j}\right]_{t}^{c}+V\left(t, S_{t-} e^{\Delta X_{t}}\right) \\
& \left.-V\left(t, S_{t-}\right)-\sum_{i=1}^{d} S_{t-}^{i}\left(e^{\Delta X_{t}^{i}}-1\right) \frac{\partial V}{\partial s_{i}}\left(t, S_{t-}\right)\right) \\
= & a(t) \mathrm{d} t+\mathrm{d} M_{t},
\end{aligned}
$$


where

$$
\begin{aligned}
a(t)= & -r e^{-r t} V+e^{-r t}\left(\frac{\partial V}{\partial t}+\sum_{i=1}^{d} \frac{\partial V}{\partial s_{i}} r S_{t-}^{i}+\frac{1}{2} \sum_{i, j=1}^{d} \mathcal{Q}_{i j} S_{t-}^{i} S_{t-}^{j} \frac{\partial^{2} V}{\partial s_{i} \partial s_{j}}\right. \\
& \left.+\int_{\mathbb{R}^{d}}\left(V\left(t, S_{t-} e^{z}\right)-V\left(t, S_{t-}\right)-\sum_{i=1}^{d} S_{t-}^{i}\left(e^{z_{i}}-1\right) \frac{\partial V}{\partial s_{i}}\left(t, S_{t-}\right)\right) v(\mathrm{~d} z)\right), \\
\mathrm{d} M_{t}= & e^{-r t}\left(\sum_{i=1}^{d} \frac{\partial V}{\partial s_{i}}\left(t, S_{t-}\right) S_{t-}^{i} \sum_{k=1}^{d} \Sigma_{i k} \mathrm{~d} W_{t}^{k}\right. \\
& \left.+\int_{\mathbb{R}^{d}}\left(V\left(t, S_{t-} e^{z}\right)-V\left(t, S_{t-}\right)\right) \tilde{J}(\mathrm{~d} t, \mathrm{~d} z)\right) .
\end{aligned}
$$

Since $g$ is Lipschitz, $V$ also is Lipschitz with respect to $s$, and $\frac{\partial V}{\partial s_{i}}$ is bounded, $i=1, \ldots, d$. With

$$
\begin{aligned}
& \mathbb{E}\left(\int_{0}^{T} \int_{\mathbb{R}^{d}}\left(V\left(t, S_{t-} e^{z}\right)-V\left(t, S_{t-}\right)\right)^{2} v(\mathrm{~d} z) \mathrm{d} t\right) \\
& \quad \lesssim \mathbb{E}\left(\int_{0}^{T} \int_{\mathbb{R}^{d}} \sum_{i=1}^{d}\left(S_{t-}^{i}\right)^{2}\left(e^{2 z_{i}}+1\right) v(\mathrm{~d} z) \mathrm{d} t\right) \\
& \quad \lesssim \sum_{i=1}^{d} \int_{\mathbb{R}}\left(e^{2 z_{i}}+1\right) v_{i}\left(\mathrm{~d} z_{i}\right) \mathbb{E}\left(\int_{0}^{T}\left(S_{t-}^{i}\right)^{2} \mathrm{~d} t\right)<\infty
\end{aligned}
$$

and

$$
\mathbb{E}\left(\int_{0}^{T}\left(S_{t-}^{i}\right)^{2}\left|\frac{\partial V}{\partial s_{i}}\left(t, S_{t-}\right)\right| \mathrm{d} t\right) \lesssim \mathbb{E}\left(\int_{0}^{T}\left(S_{t-}^{i}\right)^{2} \mathrm{~d} t\right)<\infty
$$

for $i=1, \ldots, d, M$ is a square-integrable martingale by [11, Proposition 8.6]. Therefore $\left\{e^{-r t} V_{t}-M_{t}\right\}$ is a martingale, and since $e^{-r t} V_{t}-M_{t}=\int_{0}^{t} a(\tau) \mathrm{d} \tau$ is also a continuous process with bounded variation, we have $a(t)=0$ almost surely, by [11, Proposition 8.9]. This yields the desired PIDE.

The PIDE (4.2) can further be transformed into a simpler form:

Corollary 4.3 Let $X$ be a Lévy process with state space $\mathbb{R}^{d}$, characteristic triplet $(\mathcal{Q}, v, \gamma)$, and marginal Lévy measures $v_{i}, i=1, \ldots, d$ satisfying (3.1) with $M_{i}>1$, $G_{i}>0, i=1, \ldots, d$. Furthermore, let

$$
u(\tau, x)=e^{r \tau} V\left(T-\tau, e^{x_{1}+\left(\gamma_{1}-r\right) \tau}, \ldots, e^{x_{d}+\left(\gamma_{d}-r\right) \tau}\right),
$$

where

$$
\gamma_{i}=\frac{\mathcal{Q}_{i i}}{2}+\int_{\mathbb{R}}\left(e^{z_{i}}-1-z_{i}\right) v_{i}\left(\mathrm{~d} z_{i}\right)
$$


Then $u$ satisfies the PIDE

$$
\frac{\partial u}{\partial \tau}+\mathcal{A}_{\mathrm{BS}}[u]+\mathcal{A}_{\mathrm{J}}[u]=0
$$

in $(0, T) \times \mathbb{R}^{d}$ with initial condition $u(0, x):=u_{0}$. The differential operator $\mathcal{A}_{\mathrm{BS}}$ is defined for $\varphi \in C_{0}^{2}\left(\mathbb{R}^{d}\right)$ by

$$
\mathcal{A}_{\mathrm{BS}}[\varphi]=-\frac{1}{2} \sum_{i, j=1}^{d} \mathcal{Q}_{i j} \frac{\partial^{2} \varphi}{\partial x_{i} \partial x_{j}}
$$

and the integro-differential operator $\mathcal{A}_{\mathrm{J}}$ is given by

$$
\mathcal{A}_{\mathrm{J}}[\varphi]=-\int_{\mathbb{R}^{d}}\left(\varphi(x+z)-\varphi(x)-z \cdot \nabla_{x} \varphi(x)\right) v(\mathrm{~d} z) .
$$

The initial condition is given by

$$
u_{0}=g\left(e^{x}\right):=g\left(e^{x_{1}}, \ldots, e^{x_{d}}\right) .
$$

Proof We proceed in several steps. To obtain constant coefficients, we set $x_{i}=\log s_{i}$. Furthermore, we change to time to maturity $\tau=T-t$ and set

$$
u(\tau, x)=V\left(T-\tau, e^{x_{1}}, \ldots, e^{x_{d}}\right) .
$$

The resulting differential operator is given by

$$
\mathcal{A}_{\mathrm{BS}}[\varphi]=-\frac{1}{2} \sum_{i, j=1}^{d} \mathcal{Q}_{i j} \frac{\partial^{2} \varphi}{\partial x_{i} \partial x_{j}}+\sum_{i=1}^{d}\left(\frac{1}{2} \mathcal{Q}_{i i}-r\right) \frac{\partial \varphi}{\partial x_{i}}+r \varphi,
$$

and the integro-differential operator by

$$
\mathcal{A}_{\mathrm{J}}[\varphi]=-\int_{\mathbb{R}^{d}}\left(\varphi(x+z)-\varphi(x)-\sum_{i=1}^{d}\left(e^{z_{i}}-1\right) \frac{\partial \varphi}{\partial x_{i}}(x)\right) v(\mathrm{~d} z) .
$$

The interest rate $r$ can be set to zero by transforming $u$ to $\tilde{u}$ using

$$
u(\tau, x)=e^{-r \tau} \tilde{u}(\tau, x+r \tau) .
$$

Furthermore, the integro-differential operator can be rewritten as

$$
\mathcal{A}_{\mathrm{J}}[\varphi]=-\int_{\mathbb{R}^{d}}\left(\varphi(x+z)-\varphi(x)-z \cdot \nabla_{x} \varphi(x)\right) v(\mathrm{~d} z)+\tilde{\gamma} \cdot \nabla_{x} \varphi(x),
$$

where the coefficients of the drift vector $\tilde{\gamma}$ are given by

$$
\tilde{\gamma}_{i}=\int_{\mathbb{R}}\left(e^{z_{i}}-1-z_{i}\right) v_{i}\left(\mathrm{~d} z_{i}\right), \quad i=1, \ldots, d .
$$


We remove the drift in the integro-differential and in the diffusion operator by setting

$$
u(\tau, x)=\check{u}\left(\tau, x_{1}-\gamma_{1} \tau, \ldots, x_{d}-\gamma_{d} \tau\right) .
$$

\subsection{Barrier contracts}

In this section we derive the PIDE for knock-out barrier options (see, e.g., [11, Sect. 12.1.2] for the one-dimensional case). The prices of the corresponding knock-in and other barrier contracts with the same barrier can then be obtained using superposition and linearity arguments (see, e.g., [4, Sect. 6]). Let $G \subset \mathbb{R}_{\geq 0}^{d}$ be an open subset, and let $\tau_{G}=\inf \left\{t \geq 0 \mid X_{t} \in G^{c}\right\}$ be the first hitting time of the complement set $G^{c}=\mathbb{R}^{d} \backslash G$ by $X$. Then the price of a knock-out barrier option with payoff $g$ is given by

$$
V_{G}(t, s)=\mathbb{E}\left(e^{-r(T-t)} g\left(S_{T}\right) 1_{\left\{T<\tau_{G}\right\}} \mid S_{t}=s\right) .
$$

If $V_{G}$ is sufficiently smooth, it can be computed as the solution of a PIDE.

Theorem 4.4 Assume that $V_{G}(t, s)$ in (4.7) satisfies

$$
V_{G}(t, s) \in C^{1,2}\left((0, T) \times \mathbb{R}_{>0}^{d}\right) \cap C^{0}\left([0, T] \times \mathbb{R}_{\geq 0}^{d}\right) .
$$

Then $V_{G}(t, s)$ satisfies the PIDE

$$
\begin{aligned}
& \frac{\partial V_{G}}{\partial t}(t, s)+\frac{1}{2} \sum_{i, j=1}^{d} s_{i} s_{j} \mathcal{Q}_{i j} \frac{\partial^{2} V_{G}}{\partial s_{i} \partial s_{j}}+r \sum_{i=1}^{d} s_{i} \frac{\partial V_{G}}{\partial s_{i}}(t, s)-r V_{G}(t, s) \\
& \quad+\int_{\mathbb{R}^{d}}\left(V_{G}\left(t, s e^{z}\right)-V_{G}(t, s)-\sum_{i=1}^{d} s_{i}\left(e^{z_{i}}-1\right) \frac{\partial V_{G}}{\partial s_{i}}(t, s)\right) v(\mathrm{~d} z)=0
\end{aligned}
$$

on $(0, T) \times G$, where the terminal condition is given by

$$
V_{G}(T, s)=g(s) \quad \forall s \in G,
$$

and the "boundary" condition reads

$$
V_{G}(t, s)=0, \quad \text { for all }(t, s) \in(0, T) \times G^{c} .
$$

Proof Define the deterministic function $\widetilde{g}(s):=g(s) 1_{\{s \in G\}}$ and consider the European vanilla-type price function

$$
\widetilde{V}(t, s)=\mathbb{E}\left(e^{-r(T-t)} \widetilde{g}\left(S_{T \wedge \tau_{G}}\right) \mid S_{t}=s\right) .
$$

Since $S$ is a strong Markov process, we have $V_{G}\left(t, S_{t}\right)=\widetilde{V}\left(t, S_{t}\right)$ for all $t \leq T \wedge \tau_{G}$. Thus, applying the Itô formula as in the proof of Theorem 4.2 one obtains that $V_{G}$ satisfies (4.9) on $(0, T) \times G$. By definition there also holds $V_{G}\left(t, S_{t}\right)=0$ for all $\left(t, S_{t}\right) \in(0, T) \times G^{c}$. 
Remark 4.5 Note that in contrast to plain European vanilla contracts, the price $V_{G}$ of a barrier contract does not satisfy the smoothness condition (4.8) for general Lévy models. The validity of (4.8) can however be shown in case the process $X$ admits a nonvanishing diffusion component, i.e., $\mathcal{Q}>0$. Also for market models satisfying the ACP condition of [38, Definition 41.11], Theorem 4.4 can be shown to hold, see [4].

\subsection{American contracts}

Using the notation of the previous sections, we now consider an American option with maturity $T<\infty$ and Lipschitz-continuous payoff $g(s)$. Its price $V_{\mathrm{A}}(t, s)$ is given by the optimal stopping problem

$$
V_{\mathrm{A}}(t, s)=\sup _{\tau \in \mathcal{T}_{t, T}} \mathbb{E}\left(e^{-r(T-\tau)} g\left(S_{\tau}\right) \mid S_{t}=s\right),
$$

where $\mathcal{T}_{t, T}$ denotes the set of all stopping times with values between $t$ and $T$.

In $[33,34]$ it is shown how the price $V_{\mathrm{A}}(t, s)$ can be characterized as the viscosity solution of a corresponding Bellman equation (for details on viscosity solutions, we refer to, e.g., [15] and the original sources [14, 40, 42]):

Theorem 4.6 The price $V_{\mathrm{A}}(t, s)$ of an American option defined in (4.10) is a viscosity solution of

$$
\min \left\{\begin{array}{l}
r V_{\mathrm{A}}(t, s)-\frac{\partial V_{\mathrm{A}}}{\partial t}(t, s)-\frac{1}{2} \sum_{i, j=1}^{d} s_{i} s_{j} \mathcal{Q}_{i j} \frac{\partial^{2} V_{\mathrm{A}}}{\partial s_{i} \partial s_{j}}-r \sum_{i=1}^{d} s_{i} \frac{\partial V_{\mathrm{A}}}{\partial s_{i}}(t, s) \\
\quad-\int_{\mathbb{R}^{d}}\left(V_{\mathrm{A}}\left(t, s e^{z}\right)-V_{\mathrm{A}}(t, s)-\sum_{i=1}^{d} s_{i}\left(e^{z_{i}}-1\right) \frac{\partial V_{\mathrm{A}}}{\partial s_{i}}(t, s)\right) v(\mathrm{~d} z), \\
V_{\mathrm{A}}(t, s)-g(s)
\end{array}\right\}=0 .
$$

If $V_{\mathrm{A}}(t, s)$ is uniformly continuous and

$$
\sup _{[0, T] \times \mathbb{R}_{>0}^{d}} \frac{V_{\mathrm{A}}(t, s)}{1+s}<\infty
$$

this solution is unique.

Proof The existence of the viscosity solution follows from [34, Theorem 3.1], and its uniqueness is ensured by [34, Theorem 4.1] and [40].

Analogously to Corollary 4.3 , by setting

$$
\begin{gathered}
u_{\mathrm{A}}(\tau, x)=e^{r \tau} V_{\mathrm{A}}\left(T-\tau, e^{x_{1}+\left(\gamma_{1}-r\right) \tau}, \ldots, e^{x_{d}+\left(\gamma_{d}-r\right) \tau}\right), \quad \tau \in[0, T], x \in \mathbb{R}^{d}, \\
\widetilde{g}_{\tau}(x)=g\left(e^{x_{1}+\left(\gamma_{1}-r\right) \tau}, \ldots, e^{x_{d}+\left(\gamma_{d}-r\right) \tau}\right), \quad \tau \in[0, T], x \in \mathbb{R}^{d},
\end{gathered}
$$

with $\gamma_{i}, i=1, \ldots, d$, as in (4.3), the Bellman equation (4.11) can equivalently be restated as the linear complementarity problem

$$
\frac{\partial u_{\mathrm{A}}}{\partial \tau}(\tau, x)+\mathcal{A}_{\mathrm{BS}}\left[u_{\mathrm{A}}\right](\tau, x)+\mathcal{A}_{\mathrm{J}}\left[u_{\mathrm{A}}\right](\tau, x) \leq 0,
$$




$$
\begin{aligned}
& u_{\mathrm{A}}(\tau, x)-e^{r \tau} \widetilde{g}_{\tau}(x) \geq 0, \\
& \left(\frac{\partial u_{\mathrm{A}}}{\partial \tau}(\tau, x)+\mathcal{A}_{\mathrm{BS}}\left[u_{\mathrm{A}}\right](\tau, x)+\mathcal{A}_{\mathrm{J}}\left[u_{\mathrm{A}}\right](\tau, x)\right)\left(u_{\mathrm{A}}(\tau, x)-e^{r \tau} \widetilde{g}_{\tau}(\tau, x)\right)=0
\end{aligned}
$$

on $[0, T] \times \mathbb{R}^{d}$ with $\mathcal{A}_{\mathrm{BS}}$ and $\mathcal{A}_{\mathrm{J}}$ defined in (4.4) and (4.5). As in (4.6), the initial condition is given by $u_{\mathrm{A}, 0}=g\left(e^{x}\right)$, i.e., $u_{\mathrm{A}, 0}=u_{0}$.

\subsection{Variational formulation}

For $u, v \in C_{0}^{\infty}\left(\mathbb{R}^{d}\right)$, we associate with $\mathcal{A}_{\mathrm{BS}}$ the bilinear form

$$
\mathcal{E}_{\mathrm{BS}}(u, v)=\frac{1}{2} \sum_{i, j=1}^{d} \mathcal{Q}_{i j} \int_{\mathbb{R}^{d}} \frac{\partial u}{\partial x_{i}}(x) \frac{\partial v}{\partial x_{j}}(x) \mathrm{d} x .
$$

To the jump part $\mathcal{A}_{\mathrm{J}}$ we associate the bilinear canonical jump form

$$
\mathcal{E}_{\mathrm{J}}^{\mathrm{C}}(u, v)=-\int_{\mathbb{R}^{d}} \int_{\mathbb{R}^{d}}\left(u(x+z)-u(x)-\sum_{i=1}^{d} z_{i} \frac{\partial u}{\partial x_{i}}(x)\right) v(x) \mathrm{d} x v(\mathrm{~d} z)
$$

and set

$$
\mathcal{E}(u, v)=\mathcal{E}_{\mathrm{BS}}(u, v)+\mathcal{E}_{\mathrm{J}}^{\mathrm{C}}(u, v) .
$$

We can now formulate the realization of the abstract problem (2.8) for European contracts with $\mathcal{V}=\mathcal{D}(\mathcal{E})$ and $\mathcal{H}=L^{2}\left(\mathbb{R}^{d}\right)$ as

$$
\begin{aligned}
& \text { find } u \in L^{2}((0, T) ; \mathcal{D}(\mathcal{E})) \cap H^{1}\left((0, T) ; \mathcal{D}(\mathcal{E})^{*}\right) \text { such that } \\
& \left\langle\frac{\partial u}{\partial \tau}, v\right\rangle_{\mathcal{D}(\mathcal{E})^{*}, \mathcal{D}(\mathcal{E})}+\mathcal{E}(u, v)=0, \quad \tau \in(0, T), \forall v \in \mathcal{D}(\mathcal{E}), \\
& u(0)=u_{0},
\end{aligned}
$$

where $u_{0}$ is defined as in (4.6). Furthermore, if the solution $u_{\mathrm{A}}$ of (4.13) satisfies

$$
u_{\mathrm{A}} \in L^{2}((0, T) ; \mathcal{D}(\mathcal{E})) \cap H^{1}\left((0, T) ; \mathcal{D}(\mathcal{E})^{*}\right),
$$

it can be identified with the solution of the following realization of the abstract variational inequality (2.11)-(2.12):

$$
\begin{aligned}
& \text { find } u_{\mathrm{A}} \in L^{2}((0, T) ; \mathcal{D}(\mathcal{E})) \cap H^{1}\left((0, T) ; \mathcal{D}(\mathcal{E})^{*}\right) \text { s.t. } u_{\mathrm{A}} \in \mathcal{D}\left(\phi_{\tau}\right) \text { a.e. in }(0, T), \\
& \left\langle\frac{\partial u_{\mathrm{A}}}{\partial \tau}, v-u_{\mathrm{A}}\right\rangle_{\mathcal{D}(\mathcal{E})^{*}, \mathcal{D}(\mathcal{E})}+\mathcal{E}\left(u_{\mathrm{A}}, v-u_{\mathrm{A}}\right)-\phi_{\tau}(u)+\phi_{\tau}(v) \geq 0,
\end{aligned}
$$

for all $v \in \mathcal{D}\left(\phi_{\tau}\right)$, a.e. in $(0, T)$, and $u_{\mathrm{A}}(0)=u_{0}$,

with $\phi_{\tau}:=I_{\mathcal{K}_{\tau}}$ as in (2.10) and convex sets

$$
\mathcal{K}_{\tau}:=\left\{v \in \mathcal{D}(\mathcal{E}): v \geq e^{r \tau} \widetilde{g}_{\tau}\right\} \subset \mathcal{D}(\mathcal{E}), \quad \tau \in(0, T),
$$


where $\widetilde{g}_{\tau}: \mathbb{R}^{d} \rightarrow \mathbb{R}$ is given by (4.12). As illustrated in Sect. 2.3 , the variational problem (4.16) in weak form reads

$$
\begin{aligned}
& \text { find } u_{\mathrm{A}} \in L^{\infty}((0, T) ; \mathcal{D}(\mathcal{E})) \cap H^{1}\left((0, T) ; \mathcal{D}(\mathcal{E})^{*}\right) \text { s.t. } u_{\mathrm{A}} \in \mathcal{D}(\Phi) \text { a.e. in }(0, T) \\
& \int_{0}^{T}\left\langle\frac{\partial v}{\partial \tau}(\tau)+(\mathcal{A}+\lambda) u_{\mathrm{A}}(\tau)-\lambda v(\tau), u_{\mathrm{A}}(\tau)-v(\tau)\right\rangle e^{-2 \lambda \tau} \mathrm{d} \tau+\Phi\left(u_{\mathrm{A}}\right)-\Phi(v) \\
& \quad \leq \frac{1}{2}\left\|u_{0}-v(0)\right\|_{\mathcal{H}}^{2} \quad \text { for all } v \in \mathcal{D}(\Phi) \text { with } \frac{\partial v}{\partial \tau} \in L^{2}\left(0, T ; \mathcal{V}^{*}\right)
\end{aligned}
$$

Here $\Phi$ and $\mathcal{D}(\Phi)$ are depending on $\phi_{\tau}$ as defined in Sect. 2.3.

Remark 4.7 In (4.15)-(4.17), it is required that $u_{0} \in \mathcal{H}=L^{2}\left(\mathbb{R}^{d}\right)$, which implies a growth condition on the payoff $g$. In Sect. 4.5 we reformulate the problem on a bounded domain where this condition can be weakened. The weaker growth condition is given explicitly in (4.21).

The well-posedness of (4.15) and (4.17) is ensured by the following:

Theorem 4.8 Let $X$ be a Lévy process with state space $\mathbb{R}^{d}$, characteristic triplet $(\mathcal{Q}, v, \gamma)$, and Dirichlet form $\mathcal{E}(\cdot, \cdot)$. Assume that either $\mathcal{Q}>0$ or Assumptions 3.3 and 3.8 hold in conjunction with $\gamma=0$. Then, the variational equation (4.15) and the weak variational inequality (4.17) with $u_{0} \in L^{2}\left(\mathbb{R}^{d}\right)$ admit a unique solution in $\mathcal{D}(\mathcal{E})$.

For $\mathcal{Q}>0$, we have $\mathcal{D}(\mathcal{E})=H^{1}\left(\mathbb{R}^{d}\right)$, and for $\mathcal{Q}=0$, one obtains $\mathcal{D}(\mathcal{E})=H^{\left(Y_{1} / 2, \ldots, Y_{d} / 2\right)}\left(\mathbb{R}^{d}\right)$, where

$$
H^{\left(s_{1}, \ldots, s_{d}\right)}\left(\mathbb{R}^{d}\right)=\left\{u \in L^{2}\left(\mathbb{R}^{d}\right): \int_{\mathbb{R}^{d}} \sum_{j=1}^{d}\left(1+\xi_{j}^{2}\right)^{s_{j}}|\widehat{u}(\xi)|^{2} \mathrm{~d} \xi<\infty\right\}
$$

is an anisotropic Sobolev space.

Proof Since a Lévy process $X$ is stationary, its infinitesimal generator is translation invariant. We also have with Theorem 3.10 that the characteristic exponent $\psi$ of $X$ satisfies the sector condition (3.3). Therefore, the bilinear form $\mathcal{E}(u, v)$ is a Dirichlet form and, by [22, Example 4.7.32], it can be written as

$$
|\mathcal{E}(u, v)|=(2 \pi)^{d}\left|\int_{\mathbb{R}^{d}} \psi(\xi) \widehat{u}(\xi) \overline{\widehat{v}(\xi)} \mathrm{d} \xi\right|
$$

By Theorem 2.10, for the existence and uniqueness of a solution of (4.15), we need to show that $\mathcal{E}(\cdot, \cdot)$ satisfies the continuity condition (2.6) and the Gårding inequality (2.7). 
First, consider the case $\mathcal{Q}=0$. By Propositions 3.5 and 3.9, there exist some constants $C_{1}, C_{2}, C_{3}>0$ such that

$$
\begin{aligned}
& \Re \psi(\xi) \geq C_{1} \sum_{j=1}^{d}\left|\xi_{j}\right|^{Y_{j}}-C_{2}, \\
& |\psi(\xi)| \leq C_{3}\left(\sum_{j=1}^{d}\left|\xi_{j}\right|^{Y_{j}}+1\right) \text { for all } \xi \in \mathbb{R}^{d} .
\end{aligned}
$$

Therefore, the continuity of $\mathcal{E}(\cdot, \cdot)$ is ensured by

$$
\begin{aligned}
|\mathcal{E}(u, v)| & =\left|\int_{\mathbb{R}^{d}} \psi(\xi) \widehat{u}(\xi) \overline{\widehat{v}(\xi)} \mathrm{d} \xi\right| \\
\leq & C_{3} \int_{\mathbb{R}^{d}}\left(1+\sum_{i=1}^{d}\left|\xi_{i}\right|^{Y_{i}}\right) \widehat{u}(\xi) \overline{\widehat{v}(\xi)} \mathrm{d} \xi \\
\leq & \widetilde{C}_{3} \int_{\mathbb{R}^{d}} \sum_{i=1}^{d}\left(1+\left|\xi_{i}\right|^{2}\right)^{Y_{i} / 2} \widehat{u}(\xi) \widehat{\widehat{v}(\xi)} \mathrm{d} \xi \\
\leq & \widetilde{C}_{3} \sqrt{\int_{\mathbb{R}^{d}} \sum_{i=1}^{d}\left(1+\left|\xi_{i}\right|^{2}\right)^{Y_{i} / 2}|\widehat{u}(\xi)|^{2} \mathrm{~d} \xi} \\
& \times \sqrt{\int_{\mathbb{R}^{d}} \sum_{i=1}^{d}\left(1+\left|\xi_{i}\right|^{2}\right)^{Y_{i} / 2}|\widehat{v}(\xi)|^{2} \mathrm{~d} \xi} \\
& \lesssim\|u\|_{H^{\left(Y_{1} / 2, \ldots, Y_{d} / 2\right)}\left(\mathbb{R}^{d}\right)}\|v\|_{H^{\left(Y_{1} / 2, \ldots, Y_{d} / 2\right)}\left(\mathbb{R}^{d}\right)}
\end{aligned}
$$

where we used $\sum_{i=1}^{d}\left(1+\left|\xi_{i}\right|^{2}\right)^{Y_{i} / 2} \sim\left(1+\sum_{i=1}^{d}\left|\xi_{i}\right|^{Y_{i}}\right)$. Furthermore, for the Gårding inequality, one finds

$$
\begin{aligned}
\mathcal{E}(u, u) & =\int_{\mathbb{R}^{d}} \Re \psi(\xi)|\widehat{u}(\xi)|^{2} \mathrm{~d} \xi \\
& =\int_{\mathbb{R}^{d}}\left(C_{1}+C_{2}+\Re \psi(\xi)\right)|\widehat{u}(\xi)|^{2} \mathrm{~d} \xi-\left(C_{1}+C_{2}\right) \int_{\mathbb{R}^{d}}|\widehat{u}(\xi)|^{2} \mathrm{~d} \xi
\end{aligned}
$$

and

$$
\begin{aligned}
\int_{\mathbb{R}^{d}}\left(C_{1}+C_{2}+\Re \psi(\xi)\right)|\widehat{u}(\xi)|^{2} \mathrm{~d} \xi & \geq C_{1} \int_{\mathbb{R}^{d}}\left(1+\sum_{i=1}^{d}\left|\xi_{i}\right|^{Y_{i}}\right)|\widehat{u}(\xi)|^{2} \mathrm{~d} \xi \\
& \geq \widetilde{C}_{1} \int_{\mathbb{R}^{d}} \sum_{i=1}^{d}\left(1+\left|\xi_{i}\right|^{2}\right)^{Y_{i} / 2}|\widehat{u}(\xi)|^{2} \mathrm{~d} \xi .
\end{aligned}
$$


Theorem 2.10 therefore implies the existence and uniqueness of a solution $u \in \mathcal{D}(\mathcal{E})=H^{\left(Y_{1} / 2, \ldots, Y_{d} / 2\right)}\left(\mathbb{R}^{d}\right)$ of (4.15). One obtains the existence and uniqueness of the solution $u_{\mathrm{A}}$ of (4.17) analogously from Theorem 2.13 in conjunction with, e.g., [6, Remark 3] (to account for the smooth time-dependence of the convex set $\mathcal{K}_{\tau}$ ).

If $\mathcal{Q}>0$, one obtains the required results using the same arguments. By (3.7) and (3.8), instead of (4.18) in this case there holds

$$
\Re \psi(\xi) \gtrsim \sum_{j=1}^{d}\left|\xi_{j}\right|^{2}, \quad|\psi(\xi)| \lesssim \sum_{j=1}^{d}\left|\xi_{j}\right|^{2} \quad \text { for all }\|\xi\|_{\infty}>1
$$

and the result follows as above.

Remark 4.9 We omitted the partially degenerate case $\mathcal{Q} \neq 0$ but $\mathcal{Q} \ngtr 0$ in Theorem 4.8. Here, the domain $\mathcal{D}(\mathcal{E})$ can be obtained by writing

$$
\mathcal{Q}=\left(\sigma_{i} \sigma_{j} \rho_{i j}\right)_{1 \leq i, j \leq d}
$$

where $\rho_{i j}$ is the correlation of the Brownian motions $W_{i}$ and $W_{j}$. Suppose that $\sigma_{i}=0$ for all $i \in \mathcal{I} \subset\{1, \ldots, d\}$ and $\sigma_{j}>0$ for all $j \notin \mathcal{I}$. By [30, Sect. 9.2] the anisotropic Sobolev spaces in Theorem 4.8 possess an intersection structure

$$
H^{\left(s_{1}, \ldots, s_{d}\right)}\left(\mathbb{R}^{d}\right)=\bigcap_{j=1}^{d} H_{j}^{s_{j}}\left(\mathbb{R}^{d}\right), \quad\left(s_{1}, \ldots, s_{d}\right) \in \mathbb{R}^{d},
$$

with

$$
H_{j}^{s_{j}}\left(\mathbb{R}^{d}\right)=\left\{f \in \mathcal{S}^{\prime}\left(\mathbb{R}^{d}\right):\|f\|_{H_{j}^{s_{j}}\left(\mathbb{R}^{d}\right)}=\left\|\left(1+\xi_{j}^{2}\right)^{s_{j} / 2} \widehat{f}\right\|_{L^{2}\left(\mathbb{R}^{d}\right)}<\infty\right\}
$$

Using the above arguments, one obtains

$$
\mathcal{D}(\mathcal{E})=\bigcap_{i \in \mathcal{I}} H_{i}^{\frac{Y_{i}}{2}}\left(\mathbb{R}^{d}\right) \cap \bigcap_{j \notin \mathcal{I}} H_{j}^{1}\left(\mathbb{R}^{d}\right)
$$

Remark 4.10 For European contracts, Theorem 4.8 was already obtained in dimension $d=1$ in Matache et al. [29]. For $d>1$, Farkas et al. [20] proved Theorem 4.8 for symmetric tempered stable margins.

For the numerical implementation of (4.15), it is important to note that all integrals in (4.14) exist in the Lebesgue sense even for functions $u, v \in H^{1}\left(\mathbb{R}^{d}\right)$ with compact supports.

Proposition 4.11 If $u, v \in H^{1}\left(\mathbb{R}^{d}\right)$ with compact supports, then $\left|\mathcal{E}_{\mathrm{J}}^{\mathrm{C}}(u, v)\right|<\infty$, where the bilinear form $\mathcal{E}_{\mathrm{J}}^{\mathrm{C}}(u, v)$ is given by (4.14). 
Proof Since $\int_{\mathbb{R}^{d}}|z|^{2} v(\mathrm{~d} z)<\infty$ by Assumption 3.1, we need to show that

$$
\left|\int_{\mathbb{R}^{d}}\left(u(x+z)-u(x)-\sum_{i=1}^{d} z_{i} \frac{\partial u}{\partial x_{i}}(x)\right) v(x) \mathrm{d} x\right| \lesssim|z|^{2}\|u\|_{H^{1}\left(\mathbb{R}^{d}\right)}\|v\|_{H^{1}\left(\mathbb{R}^{d}\right)} .
$$

Using integration by parts and the Cauchy-Schwarz inequality, we have

$$
\begin{aligned}
& \left|\int_{\mathbb{R}^{d}}\left(u(x+z)-u(x)-\sum_{i=1}^{d} z_{i} \frac{\partial u}{\partial x_{i}}(x)\right) v(x) \mathrm{d} x\right| \\
& =\mid \int_{\mathbb{R}^{d}} \sum_{i=1}^{d} z_{i} \int_{0}^{1} \frac{\partial u}{\partial x_{i}}\left(x_{1}, \ldots, x_{i}+\theta_{i} z_{i}, x_{i+1}+z_{i+1}, \ldots, x_{d}+z_{d}\right) \mathrm{d} \theta_{i} v(x) \mathrm{d} x \\
& -\int_{\mathbb{R}^{d}} \sum_{i=1}^{d} z_{i} \frac{\partial u}{\partial x_{i}}(x) v(x) \mathrm{d} x \mid \\
& =\mid \int_{\mathbb{R}^{d}} \sum_{i=1}^{d} z_{i} \int_{0}^{1} u\left(x_{1}, \ldots, x_{i}+\theta_{i} z_{i}, x_{i+1}+z_{i+1}, \ldots, x_{d}+z_{d}\right) \mathrm{d} \theta_{i} \frac{\partial v}{\partial x_{i}}(x) \mathrm{d} x \\
& -\int_{\mathbb{R}^{d}} \sum_{i=1}^{d} z_{i} u(x) \frac{\partial v}{\partial x_{i}}(x) \mathrm{d} x \\
& =\mid \int_{\mathbb{R}^{d}} \sum_{i=1}^{d} \sum_{j=i+1}^{d} z_{i} z_{j} \int_{0}^{1} \int_{0}^{1} u\left(x_{1}, \ldots, x_{i}+\theta_{i} z_{i}, x_{i+1},\right. \\
& \left.\ldots, x_{j-1}, x_{j}+\theta z_{j}, x_{j+1}+z_{i+1}, \ldots, x_{d}+z_{d}\right) \mathrm{d} \theta_{j} \mathrm{~d} \theta_{i} \frac{\partial v}{\partial x_{i}}(x) \mathrm{d} x \\
& +\int_{\mathbb{R}^{d}} \sum_{i=1}^{d} z_{i}\left(\int_{0}^{1} u\left(x_{1}, \ldots, x_{i}+\theta_{i} z_{i}, \ldots, x_{d}\right) \mathrm{d} \theta_{i}-u(x)\right) \frac{\partial v}{\partial x_{i}}(x) \mathrm{d} x \\
& \lesssim \sum_{i=1}^{d} \sum_{j=i+1}^{d}\left|z_{i} z_{j}\right|\|u\|_{L^{2}\left(\mathbb{R}^{d}\right)}\|v\|_{H^{1}\left(\mathbb{R}^{d}\right)} \\
& +\left|\int_{\mathbb{R}^{d}} \sum_{i=1}^{d} z_{i}^{2} \int_{0}^{1}\left(1-\theta_{i}\right) \frac{\partial u}{\partial x_{i}}\left(x_{1}, \ldots, x_{i}+\theta_{i} z_{i}, \ldots, x_{d}\right) \mathrm{d} \theta_{i} \frac{\partial v}{\partial x_{i}}(x) \mathrm{d} x\right| \\
& \lesssim \sum_{i=1}^{d} \sum_{j=i+1}^{d}\left|z_{i} z_{j}\right|\|u\|_{L^{2}\left(\mathbb{R}^{d}\right)}\|v\|_{H^{1}\left(\mathbb{R}^{d}\right)}+\sum_{i=1}^{d} z_{i}^{2}\|u\|_{H^{1}\left(\mathbb{R}^{d}\right)}\|v\|_{H^{1}\left(\mathbb{R}^{d}\right)} \\
& \lesssim|z|^{2}\|u\|_{H^{1}\left(\mathbb{R}^{d}\right)}\|v\|_{H^{1}\left(\mathbb{R}^{d}\right)} \text {. }
\end{aligned}
$$


We can also convert the canonical form $\mathcal{E}_{\mathrm{J}}^{\mathrm{C}}(\cdot, \cdot)$ of (4.14) into the integrated jump form $\mathcal{E}_{\mathrm{J}}^{\mathrm{I}}(\cdot, \cdot)$ by using Lemma 2.5 , to get

$$
\begin{aligned}
\mathcal{E}_{\mathrm{J}}^{\mathrm{I}}(u, v)= & -\sum_{i=1}^{d} \int_{\mathbb{R}} \int_{\mathbb{R}^{d}}\left(u\left(x+z_{i}\right)-u(x)-z_{i} \frac{\partial u}{\partial x_{i}}(x)\right) v(x) k_{i}\left(z_{i}\right) \mathrm{d} x \mathrm{~d} z_{i} \\
& -\sum_{i=2}^{d} \sum_{\substack{|I|=i \\
I_{1}<\cdots<I_{i}}} \int_{\mathbb{R}^{i}} \int_{\mathbb{R}^{d}} \frac{\partial^{i} u}{\partial x^{I}}\left(x+z^{I}\right) v(x) F^{I}\left(\left(U_{j}\left(z_{j}\right)\right)_{j \in I}\right) \mathrm{d} x \mathrm{~d} z^{I} .
\end{aligned}
$$

For the integrals in (4.19) to exist, it is sufficient that $u$ has compact support and $u \in \mathcal{H}_{\text {mix }}^{1}\left(\mathbb{R}^{d}\right)=H^{1}(\mathbb{R}) \otimes \cdots \otimes H^{1}(\mathbb{R})$. Note that tensor products of one-dimensional continuous, piecewise linear finite element basis functions satisfy these requirements.

Proposition 4.12 For $u, v \in \mathcal{H}_{\text {mix }}^{1}\left(\mathbb{R}^{d}\right)$ with compact support, $\left|\mathcal{E}_{\mathrm{J}}^{\mathrm{I}}(u, v)\right|<\infty$.

Proof Analogously to Proposition 4.11, for $u, v \in H^{1}\left(\mathbb{R}^{d}\right)$ with compact support, we have

$$
\left|\int_{\mathbb{R}^{d}}\left(u\left(x+z_{i}\right)-u(x)-z_{i} \frac{\partial u}{\partial x_{i}}(x)\right) v(x) \mathrm{d} x\right| \lesssim z_{i}^{2}\|u\|_{H^{1}\left(\mathbb{R}^{d}\right)}\|v\|_{H^{1}\left(\mathbb{R}^{d}\right)}
$$

for $i=1, \ldots, d$. With

$$
\left|\int_{\mathbb{R}^{d}} \frac{\partial^{|I|} u}{\partial x^{I}}\left(x+z^{I}\right) v(x) \mathrm{d} x\right| \leq\|u\|_{\mathcal{H}_{\text {mix }}^{1}\left(\mathbb{R}^{d}\right)}\|v\|_{H^{1}\left(\mathbb{R}^{d}\right)} \quad \forall z \in \mathbb{R}^{d}, I \subset\{1, \ldots, d\}
$$

and

$$
\left|\int_{\mathbb{R}^{|I|}} F^{I}\left(\left(U_{i}\left(z_{i}\right)\right)_{i \in I}\right) \mathrm{d} z^{I}\right|<\infty \quad \forall I \subset\{1, \ldots, d\},
$$

we obtain the asserted result.

Finally, one may also split the canonical jump form $\mathcal{E}_{\mathrm{J}}^{\mathrm{C}}(\cdot, \cdot)$ defined in (4.14) into its symmetric part $\mathcal{E}_{\mathrm{J}}^{\mathrm{sym}}(\cdot, \cdot)$ and its antisymmetric part $\mathcal{E}_{\mathrm{J}}^{\mathrm{asym}}(\cdot, \cdot)$, which are defined by

$$
\begin{aligned}
\mathcal{E}_{\mathrm{J}}^{\mathrm{sym}}(u, v) & =\frac{1}{2} \int_{\mathbb{R}^{d}} \int_{\mathbb{R}^{d}}(u(x+z)-u(x))(v(x+z)-v(x)) \mathrm{d} x k^{\mathrm{sym}}(z) \mathrm{d} z, \\
\mathcal{E}_{\mathrm{J}}^{\mathrm{asym}}(u, v) & =\int_{\mathbb{R}^{d}} \int_{\mathbb{R}^{d}}\left(\frac{u(x+z)-u(x-z)}{2}-z \cdot \nabla_{x} u(x)\right) v(x) \mathrm{d} x k^{\mathrm{asym}}(z) \mathrm{d} z,
\end{aligned}
$$

with $k^{\operatorname{sym}}(z):=\frac{1}{2}(k(z)+k(-z))$ and $k^{\operatorname{asym}}(z):=\frac{1}{2}(k(z)-k(-z))$. 
Lemma 4.13 Under the assumptions of Theorem 4.8 , for $u, v \in C_{0}^{\infty}\left(\mathbb{R}^{d}\right)$, there holds

$$
\mathcal{E}_{\mathrm{J}}^{\mathrm{C}}(u, v)=\mathcal{E}_{\mathrm{J}}^{\mathrm{sym}}(u, v)+\mathcal{E}_{\mathrm{J}}^{\mathrm{asym}}(u, v)
$$

Proof The bilinear form $\mathcal{E}_{\mathrm{J}}^{\mathrm{C}}$ is a translation invariant Dirichlet form. Hence, by [22, Example 4.7.32], it can be written as

$$
\begin{aligned}
\mathcal{E}_{\mathrm{J}}^{\mathrm{C}}(u, v) & =(2 \pi)^{d} \int_{\mathbb{R}^{d}} \psi_{\mathrm{J}}(\xi) \widehat{u}(\xi) \overline{\widehat{v}(\xi)} \mathrm{d} \xi \\
& =(2 \pi)^{d} \int_{\mathbb{R}^{d}} \Re \psi_{\mathrm{J}}(\xi) \widehat{u}(\xi) \overline{\widehat{v}(\xi)} \mathrm{d} \xi+i(2 \pi)^{d} \int_{\mathbb{R}^{d}} \Im \psi_{\mathrm{J}}(\xi) \widehat{u}(\xi) \overline{\widehat{v}(\xi)} \mathrm{d} \xi,(4.20)
\end{aligned}
$$

where $\psi_{\mathrm{J}}(\xi)=\int_{\mathbb{R}^{d}}\left(1-e^{i\langle\xi, z\rangle}+i\langle\xi, z\rangle\right) v(\mathrm{~d} z)$ denotes the jump part of the Lévy symbol $\psi$ in (2.2). Recall the convolution theorem

$$
\widehat{u}(\xi) \widehat{\widehat{v}(\xi)}=(2 \pi)^{-d} \widehat{u * \widetilde{v}}(\xi), \quad \xi \in \mathbb{R}^{d},
$$

where $\tilde{v}(\cdot):=v(-\cdot)$. Denoting by $B_{\varepsilon}(0)$ the ball of radius $\varepsilon>0$ around the origin and using Plancherel's theorem, one obtains

$$
\begin{aligned}
\int_{\mathbb{R}^{d}} & \Re \psi_{\mathrm{J}}(\xi) \widehat{u}(\xi) \overline{\widehat{v}(\xi)} \mathrm{d} \xi \\
= & \lim _{\varepsilon \rightarrow 0+} \int_{\mathbb{R}^{d} \backslash B_{\varepsilon}(0)} \int_{\mathbb{R}^{d}}(1-\cos \langle\xi, z\rangle) \widehat{u}(\xi) \widehat{v}(\xi) \mathrm{d} \xi k^{\mathrm{sym}}(z) \mathrm{d} z \\
= & \lim _{\varepsilon \rightarrow 0+}(2 \pi)^{-d} \int_{\mathbb{R}^{d} \backslash B_{\varepsilon}(0)} \int_{\mathbb{R}^{d}}(u(x) v(x)-u(x+z) v(x)) \mathrm{d} x k^{\mathrm{sym}}(z) \mathrm{d} z \\
= & (2 \pi)^{-d} \int_{\mathbb{R}^{d}} \int_{\mathbb{R}^{d}}(u(x)-u(x+z)) v(x) \mathrm{d} x k^{\mathrm{sym}}(z) \mathrm{d} z \\
= & \frac{1}{2}(2 \pi)^{-d} \int_{\mathbb{R}^{d}} \int_{\mathbb{R}^{d}}(u(x)-u(x+z)) v(x) \mathrm{d} x k^{\mathrm{sym}}(z) \mathrm{d} z \\
& +\frac{1}{2}(2 \pi)^{-d} \int_{\mathbb{R}^{d}} \int_{\mathbb{R}^{d}}(u(x-z)-u(x)) v(x-z) \mathrm{d} x k^{\mathrm{sym}}(z) \mathrm{d} z \\
= & \frac{1}{2}(2 \pi)^{-d} \int_{\mathbb{R}^{d}} \int_{\mathbb{R}^{d}}(u(x)-u(x+z)) v(x) \mathrm{d} x k^{\mathrm{sym}}(z) \mathrm{d} z \\
& +\frac{1}{2}(2 \pi)^{-d} \int_{\mathbb{R}^{d}} \int_{\mathbb{R}^{d}}(u(x+z)-u(x)) v(x+z) \mathrm{d} x k^{\mathrm{sym}}(z) \mathrm{d} z \\
= & \frac{1}{2}(2 \pi)^{-d} \int_{\mathbb{R}^{d}} \int_{\mathbb{R}^{d}}(u(x)-u(x+z))(v(x)-v(x+z)) \mathrm{d} x k^{\mathrm{sym}}(z) \mathrm{d} z,
\end{aligned}
$$

where we have used that $k^{\mathrm{sym}}$ is symmetric with respect to each coordinate axis. With 
analogous arguments, one also obtains

$$
\begin{aligned}
\int_{\mathbb{R}^{d}} & \Im \psi_{\mathrm{J}}(\xi) \widehat{u}(\xi) \overline{\widehat{v}(\xi)} \mathrm{d} \xi \\
= & \int_{\mathbb{R}^{d}} \int_{\mathbb{R}^{d}}\left(\langle\xi, z\rangle-\sin \langle\xi, z\rangle \widehat{u}(\xi) \overline{\widehat{v}(\xi)} \mathrm{d} \xi k^{\mathrm{asym}}(z) \mathrm{d} z\right. \\
= & \lim _{\varepsilon \rightarrow 0+} \int_{\mathbb{R}^{d} \backslash B_{\varepsilon}(0)}\left[\int_{\mathbb{R}^{d}}\langle\xi, z\rangle \widehat{u}(\xi) \bar{v}(\xi) \mathrm{d} \xi\right. \\
& -i(2 \pi)^{-d} \int_{\mathbb{R}^{d}} \frac{\left.e^{i\langle\xi, z\rangle}-e^{-i\langle\xi, z\rangle} \widehat{2} \widehat{u}(\xi) \overline{\widehat{v}(\xi)} \mathrm{d} \xi\right] k^{\mathrm{asym}}(z) \mathrm{d} z}{2} \lim _{\varepsilon \rightarrow 0+} \int_{\mathbb{R}^{d} \backslash B_{\varepsilon}(0)}\left[i(2 \pi)^{-d} \int_{\mathbb{R}^{d}} \sum_{i=1} z_{i} \frac{\partial u}{\partial x_{i}}(x) v(x) \mathrm{d} x\right. \\
& \left.-i(2 \pi)^{-d} \int_{\mathbb{R}^{d}} \frac{u(x+z)-u(x-z)}{2} v(x) \mathrm{d} x\right] k^{\mathrm{asym}}(z) \mathrm{d} z \\
= & i(2 \pi)^{-d} \int_{\mathbb{R}^{d}} \int_{\mathbb{R}^{d}}\left(\frac{u(x-z)-u(x+z)}{2}+\sum_{i=1}^{d} z_{i} \frac{\partial u}{\partial x_{i}}(x)\right) v(x) \mathrm{d} x k^{\mathrm{asym}}(z) \mathrm{d} z .
\end{aligned}
$$

Substituting these results back into (4.20), one obtains

$$
\mathcal{E}_{\mathrm{J}}^{\mathrm{C}}(u, v)=\mathcal{E}_{\mathrm{J}}^{\mathrm{sym}}(u, v)+\mathcal{E}_{\mathrm{J}}^{\mathrm{asym}}(u, v) .
$$

\subsection{Formulation on a bounded domain}

In this section we show how one may localize the unbounded log-price space domain $\mathbb{R}^{d}$ to a bounded domain. To analyze the effect of this localization procedure on the option price, we require the following growth condition on the payoff function: There exists some $q \geq 1$ such that

$$
g(s) \lesssim\left(\sum_{i=1}^{d} s_{i}+1\right)^{q} \quad \text { for all } s \in \mathbb{R}_{\geq 0}^{d} .
$$

This condition is satisfied by all standard multiasset options like basket, maximum, or best-of options.

\subsubsection{Localization}

The unbounded log-price domain $\mathbb{R}^{d}$ of the variable $x$ is truncated to a bounded domain $G_{R} \supseteq[-R, R]^{d}$. In terms of financial modeling, this corresponds to approximating the solution $V$ of the problem (4.2) by a barrier option $V_{R}$ which is the solution of the problem (4.9), similarly for American options. In log-price terms the European and American barrier option prices are given by

$$
u_{R}(t, x)=\mathbb{E}\left(g\left(e^{X_{T}}\right) 1_{\left\{T<\tau_{G_{R}}\right\}} \mid X_{t}=x\right),
$$




$$
u_{\mathrm{A}, R}(t, x)=\sup _{\tau \in \mathcal{T}_{t, T}} \mathbb{E}\left(g\left(e^{X_{\tau}}\right) 1_{\left\{\tau<\tau_{G_{R}}\right\}} \mid X_{t}=x\right)
$$

where, for notational convenience, we have set $r=0$. We show that for semiheavy tails the solution of the localized problem converges pointwise exponentially to the solution of the original problem.

Theorem 4.14 Suppose the payoff function $g: \mathbb{R}^{d} \rightarrow \mathbb{R}$ satisfies (4.21). Let $X$ be a Lévy process with state space $\mathbb{R}^{d}$ and Lévy measure $v$ such that the marginal measures $v_{i}$ satisfy (3.1) with $M_{i}>q, G_{i}>q, i=1, \ldots, d$, with $q$ as in (4.21). Then

$$
\left|u(t, x)-u_{R}(t, x)\right|+\left|u_{\mathrm{A}}(t, x)-u_{\mathrm{A}, R}(t, x)\right| \lesssim e^{-\alpha R+\beta\|x\|_{\infty}},
$$

with $0<\alpha<\min _{i} \min \left(G_{i}, M_{i}\right)-q$ and $\beta=\alpha+q$.

Proof We only consider the American case in detail. This also implies the case of European contracts. Let $\eta_{i}(x)$ be as in (3.2) and $M_{\tau}=\sup _{s \in[t, \tau]}\left\|X_{s}\right\|_{\infty}$. Then, with (4.21),

$$
\begin{aligned}
\left|u_{\mathrm{A}}(t, x)-u_{\mathrm{A}, R}(t, x)\right| & \leq \sup _{\tau \in \mathcal{T}_{t, T}} \mathbb{E}\left(g\left(e^{X_{\tau}}\right) 1_{\left\{\tau \geq \tau_{G_{R}}\right\}} \mid X_{t}=x\right) \\
& \lesssim \sup _{\tau \in \mathcal{T}_{t, T}} \mathbb{E}\left(e^{q M_{\tau}} 1_{\left\{M_{\tau}>R\right\}} \mid X_{t}=x\right) .
\end{aligned}
$$

Using Sato [38, Theorem 25.18], it suffices to observe for $t \leq \tau \leq T$,

$$
\begin{aligned}
& \mathbb{E}\left(e^{q\left\|X_{\tau}\right\|_{\infty}} 1_{\left\{\left\|X_{\tau}\right\|_{\infty}>R\right\}} \mid X_{t}=x\right) \\
& \quad=\int_{\mathbb{R}^{d}} e^{q\|z+x\|_{\infty}} 1_{\left\{\|z+x\|_{\infty}>R\right\}} p_{\tau-t}(z) \mathrm{d} z \\
& \lesssim e^{q\|x\|_{\infty}} \sum_{i=1}^{d} \int_{\mathbb{R}^{d}} e^{q|z|_{i}} e^{-\eta_{i}(z)} 1_{\left\{\|z+x\|_{\infty}>R\right\}} e^{\eta_{i}(z)} p_{\tau-t}(z) \mathrm{d} z \\
& \lesssim e^{q\|x\|_{\infty}} \sum_{i=1}^{d} \int_{\mathbb{R}^{d}} e^{-\left(\min _{j} \min \left(\mu_{j}^{+}, \mu_{j}^{-}\right)-q\right)\left(R-\|x\|_{\infty}\right)} e^{\eta_{i}(z)} p_{\tau-t}(z) \mathrm{d} z \\
& \lesssim e^{-\alpha R+\beta\|x\|_{\infty}} \sum_{i=1}^{d} \int_{\mathbb{R}^{d}} e^{\eta_{i}(z)} p_{\tau-t}(z) \mathrm{d} z .
\end{aligned}
$$

Then the result follows from (3.2).

The domain of integration $\mathbb{R}^{d}$ of the variable $z$ in, e.g., (4.14) can also be truncated to a bounded domain $\Lambda_{B}=[-B, B]^{d}$. For this, consider the truncated Lévy measure $v_{B}=v 1_{\left\{\|z\|_{\infty} \leq B\right\}}$ and the corresponding Lévy process $X_{B}$ with characteristic triplet $\left(\mathcal{Q}, v_{B}, \gamma_{B}\right)$. Here $\gamma_{B}$ is defined such that $e^{X_{B}^{i}}$ is a martingale, $i=1, \ldots, d$. 
Denote by $\tilde{X}=X-X_{B}$ the Lévy process with characteristic triplet $(0, \widetilde{v}, \tilde{\gamma})$, where $\widetilde{v}=v 1_{\left\{\|z\|_{\infty}>B\right\}}$. Let $u_{B}, u_{\mathrm{A}, B}$ be the solution of

$$
\begin{aligned}
u_{B}(t, x) & =7 \mathbb{E}\left(g\left(e^{X_{B, T}}\right) \mid X_{B, t}=x\right), \\
u_{\mathrm{A}, B}(t, x) & =\sup _{\tau \in \mathcal{T}_{t, T}} \mathbb{E}\left(g\left(e^{X_{B, \tau}}\right) \mid X_{B, t}=x\right),
\end{aligned}
$$

where again for notational convenience, we have set $r=0$.

Theorem 4.15 Let $X$ be a Lévy process with state space $\mathbb{R}^{d}$ and Lévy measure $v$ such that the marginal measures $v_{i}$ satisfy (3.1) with $M_{i}>1, G_{i}>0, i=1, \ldots, d$. Then

$$
\left|u(t, x)-u_{B}(t, x)\right|+\left|u_{\mathrm{A}}(t, x)-u_{\mathrm{A}, B}(t, x)\right| \lesssim e^{-\alpha B+\|x\|_{\infty}}
$$

with $0<\alpha<\min _{i} \min \left(G_{i}, M_{i}-1\right)$.

Proof Since $g$ is Lipschitz and $X_{B}, \widetilde{X}$ are independent, we have

$$
\begin{aligned}
\left|u_{\mathrm{A}}(t, x)-u_{\mathrm{A}, B}(t, x)\right| & \leq \sup _{\tau \in \mathcal{T}_{t, T}}\left|\mathbb{E}\left(g\left(e^{x+X_{\tau-t}}\right)\right)-\mathbb{E}\left(g\left(e^{x+X_{B, \tau-t}}\right)\right)\right| \\
& \lesssim \sup _{\tau \in \mathcal{T}_{t, T}} \sum_{i=1}^{d} \mathbb{E}\left(\left|e^{x_{i}+X_{\tau-t}^{i}}-e^{x_{i}+X_{B, \tau-t}^{i}}\right|\right) \\
& \lesssim \sup _{\tau \in \mathcal{T}_{t, T}} \sum_{i=1}^{d} e^{\|x\|_{\infty}} \mathbb{E}\left(e^{X_{B, \tau-t}^{i}}\left|e^{\tilde{X}_{\tau-t}^{i}}-1\right|\right) \\
& \lesssim \sup _{\tau \in \mathcal{T}_{t, T}} e^{\|x\|_{\infty}} \sum_{i=1}^{d} \mathbb{E}\left(\left|e^{\widetilde{X}_{\tau-t}^{i}}-1\right|\right) .
\end{aligned}
$$

For each summand, the desired estimate now follows from [12, Proposition 4.2], and we obtain the required result.

Remark 4.16 The localization in Theorem 4.14 and the localization in Theorem 4.15 are not equivalent. The localization of the log-price domain as in Theorem 4.14 is done to obtain a bounded computational domain $G_{R}$. Therefore, in the variational formulation a Sobolev space over $G_{R}$ is introduced which can be discretized by a finite-dimensional finite element subspace. Depending on the Lévy density and the numerical method, it may additionally be necessary to truncate the integration domain as in Theorem 4.15. In these two approximations, the stochastic process $X$ is not modified-such a modification, e.g., by replacing $X$ by a killed process $X^{G}$, would change not only the domain $G$ but also the operator $\mathcal{A}$ (see, e.g., [23, Chap. 7.2] and the references therein). 


\subsubsection{Variational formulation on the bounded domain}

For any function $u$ with support in $G_{R}$, we denote by $\tilde{u}$ its extension by zero to all of $\mathbb{R}^{d}$ and define

$$
\mathcal{E}_{R}(u, v)=\mathcal{E}(\widetilde{u}, \widetilde{v}) .
$$

Thus, we obtain continuity and a Gårding inequality of $\mathcal{E}_{R}(u, v)$ on

$$
\mathcal{D}\left(\mathcal{E}_{R}\right):=\overline{\left\{\tilde{u} \mid u \in C_{0}^{\infty}\left(G_{R}\right)\right\}},
$$

where the closure is taken with respect to the norm of $\mathcal{D}(\mathcal{E})$ as given explicitly in Theorem 4.8. Now we can restate the problem (4.15) on a bounded domain as

$$
\begin{aligned}
& \text { find } u_{R} \in L^{2}\left((0, T) ; \mathcal{D}\left(\mathcal{E}_{R}\right)\right) \cap H^{1}\left((0, T) ; \mathcal{D}\left(\mathcal{E}_{R}\right)^{*}\right) \text { such that } \\
& \begin{array}{l}
\left(\frac{\partial u_{R}}{\partial \tau}, v\right)+\mathcal{E}_{R}\left(u_{R}, v\right)=0, \quad \forall \tau \in(0, T), \forall v \in \mathcal{D}\left(\mathcal{E}_{R}\right), \\
u_{R}(0)=\left.u_{0}\right|_{G_{R}},
\end{array}
\end{aligned}
$$

where $(\cdot, \cdot)$ denotes the $L^{2}$-inner product. By Theorem 4.8 , the problem $(4.22)$ is well posed in the sense that there exists a unique solution $u_{R}$ in the space $L^{2}\left((0, T) ; \mathcal{D}\left(\mathcal{E}_{R}\right)\right) \cap C^{0}\left([0, T] ; L^{2}\left(G_{R}\right)\right)$. This solution can now be approximated by a finite element Galerkin scheme.

\section{Discretization and numerical examples}

We briefly address the discretization of (4.22). For more details, we refer to [20, 28, 45] and the references therein.

\subsection{Space discretization}

Let $V_{h}$ be a one-parameter family of subspaces $V_{h} \subset \mathcal{D}\left(\mathcal{E}_{R}\right)$ with finite dimension $N_{h}=\operatorname{dim} V_{h}<\infty$. For each $t \in(0, T)$, we approximate the solution $u_{R}(t, x)$ of (4.22) by a function $u_{h}(t) \in V_{h}$. Furthermore, let $u_{h, 0} \in V_{h}$ be an approximation of $u_{0}$. Then the semidiscrete form of (4.22) is the initial-value problem

$$
\begin{aligned}
& \text { find } u_{h} \in C^{1}\left([0, T] ; V_{h}\right) \text { such that } \\
& \left(\frac{\partial u_{h}}{\partial \tau}, v_{h}\right)+\mathcal{E}_{R}\left(u_{h}, v_{h}\right)=0, \quad \forall \tau \in(0, T), \forall v_{h} \in V_{h}, \\
& u_{h}(0)=u_{h, 0},
\end{aligned}
$$

for the approximate solution function $u_{h}(t):[0, T] \rightarrow V_{h}$. Let $V_{h}$ be generated by a finite element basis $\Phi_{h}:=\left\{\phi_{h, k}: k \in \Delta_{h}\right\}$ with index set $\Delta_{h}=\left\{1, \ldots, N_{h}\right\}$. Efficient computation depends on the choice of the basis functions $\phi_{h, k}$. Here, wavelets have three main advantages. First, they allow one to break the curse of dimension by using 
sparse tensor products to obtain essentially dimension-independent complexity [44]. Second, using a multiscale compression of the jump measure of $X$, the complexity of jump models can asymptotically be reduced to Black-Scholes complexity [35-37]. Finally, wavelets provide norm equivalences in fractional-order spaces, which leads to efficient preconditioning even for pure jump operators [20].

\subsection{Time discretization}

To realize the Galerkin finite element discretization, the Dirichlet form $\mathcal{E}_{R}(\hat{A} \cdot, \hat{A} \cdot)$ must be evaluated on the basis functions of $V_{h}$, resulting in the stiffness matrix $\mathbf{A}$ given by $\mathbf{A}_{j, i}=\mathcal{E}_{R}\left(\phi_{h, i}, \phi_{h, j}\right), i, j \in \Delta_{h}$. Furthermore, discretizing in time using the backward Euler scheme with time step $\Delta t=T / M$ and time points $t_{m}=m \Delta t$, $m=0, \ldots, M, M \in \mathbb{N}$, we obtain in matrix notation the fully discrete form

$$
\begin{aligned}
& \text { find } \underline{u}_{h}^{m+1} \in \mathbb{R}^{N_{h}} \text { such that for } m=0, \ldots, M-1, \\
& \Delta t^{-1} \mathbf{M}\left(\underline{u}_{h}^{m+1}-\underline{u}_{h}^{m}\right)+\mathbf{A} \underline{u}_{h}^{m+1}=0, \\
& \underline{u}_{h}^{0}=\underline{u}_{h, 0},
\end{aligned}
$$

where $\underline{u}_{h}^{m}$ denotes the coefficient vector of $u_{h}\left(t_{m}, \cdot\right)$, and $\mathbf{M}$ the mass matrix with respect to $\Phi_{h}$. Similarly we obtain for American options a system of matrix linear complementarity problems, namely

$$
\begin{aligned}
& \text { find } \underline{u}_{h}^{m+1} \in \underline{K} \text { such that for } m=0, \ldots, M-1, \\
& \Delta t^{-1} \mathbf{M}\left(\underline{u}_{h}^{m+1}-\underline{u}_{h}^{m}\right)+\mathbf{A} \underline{u}_{h}^{m+1} \geq 0, \\
& \left(\underline{u}_{h}^{m+1}-\underline{\widetilde{g}}^{m+1}\right)^{\top}\left(\Delta t^{-1} \mathbf{M}\left(\underline{u}_{h}^{m+1}-\underline{u}_{h}^{m}\right)+\mathbf{A} \underline{u}_{h}^{m+1}\right)=0, \\
& \underline{u}_{h}^{0}=\underline{u}_{h, 0},
\end{aligned}
$$

with $\underline{K}:=\left\{\underline{v} \in \mathbb{R}^{N_{h}} \mid \underline{v} \geq \underline{\widetilde{g}}^{m+1}\right\}$, where $\underline{\widetilde{g}}^{m}$ denotes the coefficient vector of $e^{r t_{m}} \widetilde{g}_{t_{m}}$ with respect to $\Phi_{h}$.

Remark 5.1 The main numerical problem is to calculate the stiffness matrix A since the Lévy density is singular at the origin and possibly on each axis. For $d=1$, the entries can still be calculated analytically for tempered stable densities [28, 29]. For $d>1$, one has to use composite Gauss quadrature rules which combine elementary Gauss quadrature formulas on subdomains decreasing geometrically towards the singular support of the integrand. A more detailed description and a computational scheme to compute the stiffness matrix A can be found in [45].

\subsection{Impact of diffusion approximation of small jumps}

We consider a regularization of the multivariate Lévy measure where small jumps are approximated by an artificial Brownian motion [1, 10]. This Gaussian approximation is used to simulate Lévy processes $[1,10]$ or to price options using finite differences [13]. Our discretization (5.1)-(5.3) allows us to compare the error of these approximations via accurate numerical solutions of the corresponding PIDEs. 
Let $X$ be a $d$-dimensional Lévy process with characteristic triplet $(0, v, \gamma)$ where the Lévy measure $v$ satisfies (3.1). The drift $\gamma$ is chosen according to Lemma 2.1 such that $e^{X^{j}}, j=1, \ldots, d$, are martingales. The covariance matrix is given by $\mathcal{Q}=$ $\int_{\mathbb{R}^{d}} z z^{\top} v(\mathrm{~d} z)$. For $\varepsilon>0$, let $v_{\varepsilon}$ be a measure such that $v^{\varepsilon}=v-v_{\varepsilon}$ is a finite measure. We can decompose $X$ into its small and large jump parts, i.e.,

$$
X_{t}=\gamma^{\varepsilon} t+N_{t}^{\varepsilon}+X_{\varepsilon, t}=X_{t}^{\varepsilon}+X_{\varepsilon, t},
$$

where $N^{\varepsilon}$ is a compound Poisson process with jump measure $v^{\varepsilon}$. The small jump part $X_{\varepsilon}$ is independent of the process $N^{\varepsilon}$ and has covariance matrix $\mathcal{Q}_{\varepsilon}=\int_{\mathbb{R}^{d}} z z^{\top} v_{\varepsilon}(\mathrm{d} z)$. We assume that $\mathcal{Q}_{\varepsilon}$ is nonsingular. Let $\boldsymbol{\Sigma}_{\varepsilon}$ be a nonsingular matrix with $\boldsymbol{\Sigma}_{\varepsilon} \boldsymbol{\Sigma}_{\varepsilon}^{\top}=\mathcal{Q}_{\varepsilon}$. $X_{\varepsilon}$ can be approximated by a $d$-dimensional standard Brownian motion $W$ independent of $N^{\varepsilon}$. It is shown in $[1,10]$ that, under certain assumptions on $\mathcal{Q}_{\varepsilon}$, the process $\Sigma_{\varepsilon}^{-1} X_{\varepsilon}$ converges in distribution to $W$ as $\varepsilon \rightarrow 0$.

Thus, for any $\varepsilon>0$, the process $X$ can be approximated by replacing the small jumps with a Brownian motion which yields a jump-diffusion process $Z^{\varepsilon}$ given by

$$
Z_{t}^{\varepsilon}=\Sigma_{\varepsilon} W_{t}+\gamma_{Z}^{\varepsilon} t+N_{t}^{\varepsilon}
$$

The characteristic triplet of $Z^{\varepsilon}$ is $\left(\mathcal{Q}_{\varepsilon}, v^{\varepsilon}, \gamma_{Z}^{\varepsilon}\right)$ where $\gamma_{Z}^{\varepsilon}$ is again such that $e^{Z^{\varepsilon, j}}$, $j=1, \ldots, d$, are martingales. $Z^{\varepsilon}$ has the same covariance matrix as $X$. For $\varepsilon \rightarrow \infty$, we obtain a diffusion process $Z_{t}^{\infty}=\boldsymbol{\Sigma} W_{t}+\gamma_{\infty} t$ with covariance matrix $\mathcal{Q}=\boldsymbol{\Sigma} \boldsymbol{\Sigma}^{\top}$ and drift $\gamma_{\infty, j}=-\mathcal{Q}_{j j} / 2, j=1, \ldots, d$.

There are two sources of error. We have a discretization error using a mesh width $h>0$ and a modeling error using $\varepsilon>0$. To assess the impact of $\varepsilon>0$, we use the discretization (5.1)-(5.3) for $\varepsilon=0$ and $\varepsilon>0$. Here, $h$ is chosen so small that the discretization error is negligible in comparison to the truncation error.

Remark 5.2 To obtain a converging scheme for finite difference methods, $\varepsilon>0$ was chosen in [13] depending on the mesh width $h$. For a fixed mesh width $h>0$, the discretization error increases as $\varepsilon \rightarrow 0$, i.e., $\varepsilon=0$ cannot be used.

Consider a basket option $u(t, x)$ with payoff $g(x)$ where the underlying log-price processes are given by the pure jump process $X=\left(X^{1}, \ldots, X^{d}\right)^{\top}$ and correspondingly $u^{\varepsilon}(t, x)$ for the processes $Z^{\varepsilon}$. We study the absolute error $\left|u(T, x)-u^{\varepsilon}(T, x)\right|$ versus $\epsilon$. For $d=1$, it is shown in [13] that the error satisfies $\left|u(T, x)-u^{\varepsilon}(T, x)\right| \leq \varepsilon$ for tempered stable densities. The estimate does not hold for barrier options since the option price is not smooth at the boundary $\partial G$. In particular it is shown for $d=1$ that, for tempered stable densities with $1<Y<2$ and $c^{+}=c^{-}$, the derivative of the option price behaves in $\log$-prices like $|x-\log B|^{Y / 2-1}$ as $x \rightarrow \log B$ (see, e.g., [26]). Therefore, one obtains a large error at the boundary by approximating $X$ with $Z^{\varepsilon}$. Similar comments apply for American options at spots close to the exercise boundary. 

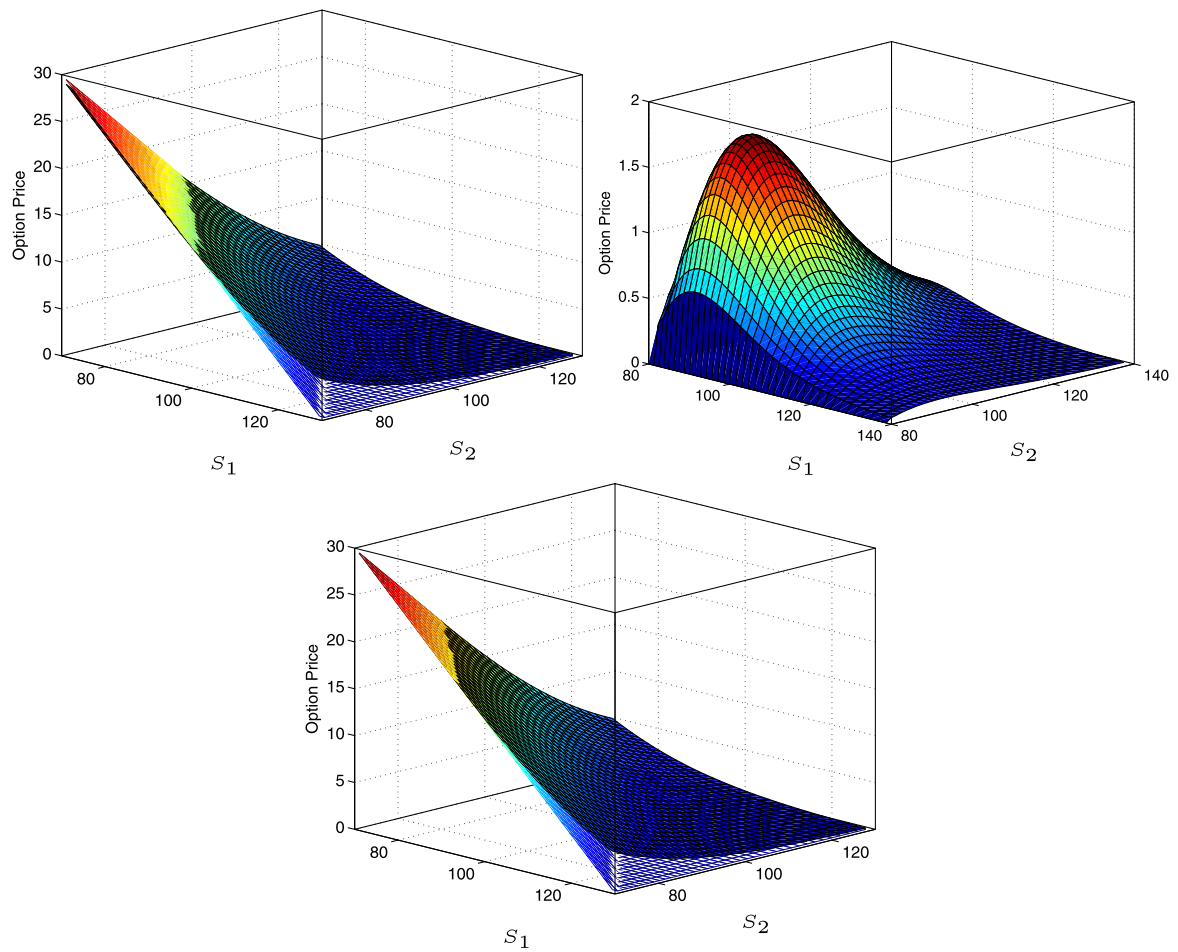

Fig. 1 European basket, barrier, and American option prices for $d=2$ with barrier $B=80$ and strike $K=100$

Example 5.3 Let $d=2$ and consider a pure jump process $(\mathcal{Q} \equiv 0)$ with two independent tempered stable marginal densities

$$
k_{i}(z)=c_{i} \frac{e^{-G_{i}|z|}}{|z|^{1+Y_{i}}} 1_{\{z<0\}}+c_{i} \frac{e^{-M_{i} z}}{z^{1+Y_{i}}} 1_{\{z>0\}}, \quad i=1,2 .
$$

We compute the price of a European basket option with payoff

$$
g\left(S_{1}, S_{2}\right)=\left(K-\frac{1}{2} S_{1}-\frac{1}{2} S_{2}\right)_{+}
$$

the price of a down-and-out barrier option with payoff $g$ and barrier $B=80$ and the price of an American option again with payoff $g$. Let the maturity be $T=0.5$, strike $K=100$, and interest rate $r=0.01$. We set $c_{1}=c_{2}=1, G_{1}=10, M_{1}=15$, $G_{2}=9, M_{2}=16, Y_{1}=0.5$, and $Y_{2}=0.7$. The option prices are shown in Fig. 1, and the relative error for approximating $X$ by $Z^{\varepsilon}$ is plotted in Fig. 2. As expected, the relative error is small for a European-style basket option. However, it is significantly higher for, e.g., a barrier option close to the barrier or for an American-style option close to the exercise boundary. 

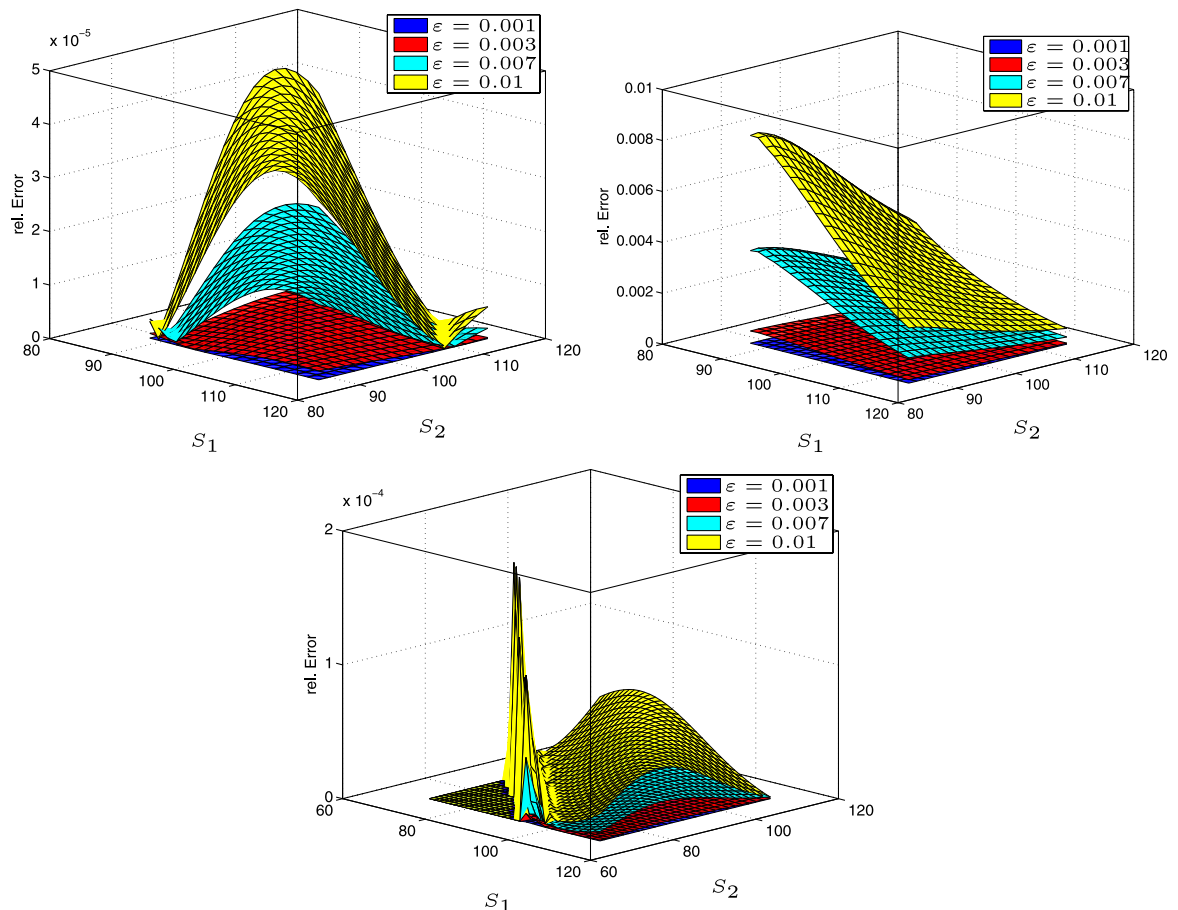

Fig. 2 Relative errors for various values of $\epsilon$ using $Z^{\varepsilon}$ from (5.5) in place of $X$ in (5.4) for a European basket, barrier, and American option for $d=2$ with barrier $B=80$ and strike $K=100$

Acknowledgements We thank Prof. R.L. Schilling who pointed out Lemma 4.13 to us. NR would like to thank the Zurich Graduate School in Mathematics (ZGSM) for financial support.

\section{Appendix: Equivalence preserving copulas}

In view of Assumption 3.8, there remains to show the equivalence preserving property of $H:=\partial_{1} \cdots \partial_{d} F$ for a large class of 1-homogeneous copulas $F$. The following lemmas provide such a class.

Lemma A.1 Suppose that $G_{1}, G_{2}: \overline{\mathbb{R}}^{d} \rightarrow \overline{\mathbb{R}}_{\geq 0}$ are two equivalence preserving functions. Then:

(i) For any $\gamma \geq 0$, the power $G_{1}(\cdot)^{\gamma}: \overline{\mathbb{R}}^{d} \rightarrow \overline{\mathbb{R}}_{\geq 0}$ is equivalence preserving on $\overline{\mathbb{R}}^{d}$.

(ii) The product $G_{1} G_{2}: \overline{\mathbb{R}}^{d} \rightarrow \overline{\mathbb{R}}_{\geq 0}$ is equivalence preserving on $\overline{\mathbb{R}}^{d}$.

(iii) The quotient $G_{1} / G_{2}: \overline{\mathbb{R}}^{d} \rightarrow \overline{\mathbb{R}}_{\geq 0}$ is equivalence preserving on any subset $\mathcal{J} \subset \mathbb{R}^{d}$ such that $\overline{\mathcal{J}}$ does not contain any poles of $G_{1} / G_{2}$.

Proof The claims follow directly from Definition 3.7. 
Lemma A.2 Consider any quasi-polynomial $P: \mathbb{R}^{d} \rightarrow \mathbb{R}_{\geq 0}$ of the form

$$
P\left(x_{1}, \ldots, x_{d}\right)=\sum_{i_{1}, \ldots, i_{d}=0}^{N} \alpha_{i_{1}, \ldots, i_{d}}\left|x_{1}\right|^{\beta_{i_{1}}} \ldots\left|x_{d}\right|^{\beta_{i_{d}}}
$$

with coefficients $\alpha_{i_{1}, \ldots, i_{d}} \geq 0$ and $\beta_{i_{k}} \geq 0$. Then $P$ is an equivalence-preserving function.

Proof Let $\mathcal{I} \subset \mathbb{R}$ and consider two families of equivalent functions $f_{i} \sim g_{i}$, $i=1, \ldots, d$, on $\mathcal{I}$. There exist constants $c_{i}, d_{i}>0$ such that

$$
c_{i}\left|f_{i}(x)\right| \leq\left|g_{i}(x)\right| \leq d_{i}\left|f_{i}(x)\right| \text { for all } x \in \mathcal{I}, i=1, \ldots, d .
$$

Thus, for any $x=\left(x_{1}, \ldots, x_{d}\right) \in \mathcal{I}^{d}$, there holds

$$
\begin{aligned}
P\left(g_{1}\left(x_{1}\right), \ldots, g_{d}\left(x_{d}\right)\right) & =\sum_{i_{1}, \ldots, i_{d}=0}^{N} d_{i_{1}}^{\beta_{i_{1}}^{1}} \cdots d_{i_{d}}^{\beta_{i_{d}}^{1}} \alpha_{i_{1}, \ldots, i_{d}}\left|f_{1}\left(x_{1}\right)^{\beta_{i_{1}}} \cdots f_{d}\left(x_{d}\right)^{\beta_{i_{d}}}\right| \\
& \leq \max _{0 \leq i_{1}, \ldots, i_{d} \leq N_{1}}\left\{d_{i_{1}}^{\beta_{i_{1}}^{1}} \cdots d_{i_{d}}^{\beta_{i_{d}}^{1}}\right\} P\left(f_{1}\left(x_{1}\right), \ldots, f_{d}\left(x_{d}\right)\right) \\
& =: D P\left(f_{1}\left(x_{1}\right), \ldots, f_{d}\left(x_{d}\right)\right) .
\end{aligned}
$$

Analogously one obtains that there exists some $C>0$ such that

$$
C P\left(f_{1}\left(x_{1}\right), \ldots, f_{d}\left(x_{d}\right)\right) \leq P\left(g_{1}\left(x_{1}\right), \ldots, g_{d}\left(x_{d}\right)\right) .
$$

Corollary A.3 For any $\theta>0$, the Clayton Lévy copula $F$ of Example 2.8 satisfies Assumption 3.8.

Proof Clearly, $F$ is 1-homogeneous, and $H:=\partial_{1} \cdots \partial_{d} F$ exists. There holds

$$
F\left(x_{1}, \ldots, x_{d}\right)=\frac{P_{1}\left(x_{1}, \ldots, x_{d}\right)^{\gamma_{1}}}{P_{2}\left(x_{1}, \ldots, x_{d}\right)^{\gamma_{2}}},
$$

where $\gamma_{1}, \gamma_{2} \geq 0$, and $P_{1}, P_{2}: \mathbb{R}^{d} \rightarrow \mathbb{R}_{\geq 0}$ are two quasi-polynomials of the form (A.1). Due to the polynomial structure, an analogous representation naturally holds for $H$. Thus, by Lemma A.1, the derivative $H$ is equivalence preserving.

\section{References}

1. Asmussen, S., Rosiński, J.: Approximations of small jumps of Lévy processes with a view towards simulation. J. Appl. Probab. 38, 482-493 (2001)

2. Baiocchi, C.: Discretization of evolution variational inequalities. In: Colombini, F., Marino, A., Modica, L., Spagnolo, S. (eds.) Partial Differential Equations and the Calculus of Variations. Progr. Nonlinear Differential Equations Appl., vol. I, pp. 59-92. Birkhäuser, Boston (1989)

3. Berg, C., Forst, G.: Non-symmetric translation invariant Dirichlet forms. Invent. Math. 21, 199-212 (1973) 
4. Boyarchenko, S., Levendorskiı̌, S.: Barrier options and touch-and-out options under regular Lévy processes of exponential type. Ann. Appl. Probab. 12, 1261-1298 (2002)

5. Brézis, H.: Problèmes unilatéraux. J. Math. Pures Appl. 51, 1-168 (1972)

6. Brézis, H.: Un problème d'evolution avec contraintes unilatérales dépendant du temps. C. R. Acad. Sci. Paris Sér. A-B 274, A310-A312 (1972)

7. Briani, M., La Chioma, C., Natalini, R.: Convergence of numerical schemes for viscosity solutions to integro-differential degenerate parabolic problems arising in financial theory. Numer. Math. 98, 607-646 (2004)

8. Carr, P., Madan, D.B.: Option pricing and the fast Fourier transform. J. Comput. Financ. 2(4), 61-73 (1999)

9. Carr, P., Geman, H., Madan, D.B., Yor, M.: The fine structure of assets returns: an empirical investigation. J. Bus. 75, 305-332 (2002)

10. Cohen, S., Rosiński, J.: Gaussian approximation of multivariate Lévy processes with applications to simulation of tempered stable processes. Bernoulli 13, 195-210 (2007)

11. Cont, R., Tankov, P.: Financial Modelling with Jump Processes. Chapman \& Hall/CRC Financial Mathematics Series. Chapman \& Hall/CRC Press, London/Boca Raton (2004)

12. Cont, R., Voltchkova, E.: A finite difference scheme for option pricing in jump diffusion and exponential Lévy models. SIAM J. Numer. Anal. 43, 1596-1626 (2005)

13. Cont, R., Voltchkova, E.: Integro-differential equations for option prices in exponential Lévy models. Finance Stoch. 9, 299-325 (2005)

14. Crandall, M.G., Lions, P.-L.: Viscosity solutions of Hamilton-Jacobi equations. Trans. Am. Math. Soc. 277, 1-42 (1983)

15. Crandall, M.G., Ishii, H., Lions, P.-L.: User's guide to viscosity solutions of second order partial differential equations. Bull. Am. Math. Soc. (N.S.) 27, 1-67 (1992)

16. Dappa, H.: Quasiradiale Fouriermultiplikatoren. Ph.D. Thesis, Darmstadt, 1982

17. Delbaen, F., Schachermayer, W.: The fundamental theorem of asset pricing for unbounded stochastic processes. Math. Ann. 312, 215-250 (1998)

18. Delbaen, F., Grandits, P., Rheinländer, T., Samperi, D., Schweizer, M., Stricker, C.: Exponential hedging and entropic penalties. Math. Financ. 12, 99-123 (2002)

19. Eberlein, E., Jacod, J.: On the range of options prices. Finance Stoch. 1, 131-140 (1997)

20. Farkas, W., Reich, N., Schwab, C.: Anisotropic stable Lévy copula processes-analytical and numerical aspects. Math. Models Methods Appl. Sci. 17, 1405-1443 (2007)

21. Glowinski, R., Lions, J.-L., Trémolières, R.: Numerical Analysis of Variational Inequalities. Studies in Mathematics and Its Applications, vol. 8. North-Holland, Amsterdam (1981). Translated from the French

22. Jacob, N.: Pseudo Differential Operators and Markov Processes. Vol. I. Fourier Analysis and Semigroups. Imperial College Press, London (2001)

23. Jacob, N.: Pseudo Differential Operators and Markov Processes. Vol. III. Markov Processes and Applications. Imperial College Press, London (2005)

24. Jacod, J., Shiryaev, A.N.: Limit Theorems for Stochastic Processes, 2nd edn. Grundlehren der Mathematischen Wissenschaften, vol. 288. Springer, Berlin (2003)

25. Kallsen, J., Tankov, P.: Characterization of dependence of multidimensional Lévy processes using Lévy copulas. J. Multivar. Anal. 97, 1551-1572 (2006)

26. Kudryavtsev, O., Levendorskiı̌, S.Z.: Fast and accurate pricing of barrier options under Lévy processes. Finance Stoch. 13, 531-562 (2009)

27. Lions, J.-L., Magenes, E.: Problèmes aux Limites Non Homogènes et Applications, vol. 1. Travaux et Recherches Mathématiques, vol. 17. Dunod, Paris (1968)

28. Matache, A.-M., Nitsche, P.-A., Schwab, C.: Wavelet Galerkin pricing of American options on Lévy driven assets. Quant. Financ. 5, 403-424 (2005)

29. Matache, A.-M., von Petersdorff, T., Schwab, C.: Fast deterministic pricing of options on Lévy driven assets. Math. Model. Numer. Anal. 38, 37-71 (2004)

30. Nikol'skiŭ, S.M.: Approximation of Functions of Several Variables and Imbedding Theorems. Grundlehren der Mathematischen Wissenschaften, vol. 205. Springer, New York (1975)

31. Nochetto, R.H., Savaré, G., Verdi, C.: A posteriori error estimates for variable time-step discretizations of nonlinear evolution equations. Commun. Pure Appl. Math. 53, 525-589 (2000)

32. Papapantoleon, A.: Applications of semimartingales and Lévy processes in finance: duality and valuation. Ph.D. Thesis, University of Freiburg, 2006. http://www.freidok.uni-freiburg.de/volltexte/2919/ pdf/thesis.pdf 
33. Pham, H.: Optimal stopping, free boundary, and American option in a jump-diffusion model. Appl. Math. Optim. 35, 145-164 (1997)

34. Pham, H.: Optimal stopping of controlled jump diffusion processes: a viscosity solution approach. J. Math. Syst. Estim. Control 8, 1-27 (1998), (electronic)

35. Reich, N.: Wavelet compression of anisotropic integrodifferential operators on sparse tensor product spaces. Ph.D. Thesis, ETH Zürich, 2008. http://e-collection.ethbib.ethz.ch/eserv/eth:30174/eth30174-02.pdf

36. Reich, N.: Wavelet compression of anisotropic integrodifferential operators on sparse tensor product spaces. Research report 2008-26, Seminar for Applied Mathematics, ETH Zürich, 2008. http://www. sam.math.ethz.ch/reports/2008/26

37. Reich, N.: Wavelet compression of integral operators on sparse tensor spaces-construction, consistency and asymptotically optimal complexity. Research report 2008-24, Seminar for Applied Mathematics, ETH Zürich, 2008. http://www.sam.math.ethz.ch/reports/2008/24

38. Sato, K.: Lévy Processes and Infinitely Divisible Distributions. Cambridge Studies in Advanced Mathematics, vol. 68. Cambridge University Press, Cambridge (1999)

39. Savaré, G.: Weak solutions and maximal regularity for abstract evolution inequalities. Adv. Math. Sci. Appl. 6, 377-418 (1996)

40. Sayah, A.: Equation d'Hamilton-Jacobi du premier ordre avec terme intégro-différentiel: Parties i et ii. Commun. Partial Differ. Equ. 16, 1057-1074 (1991)

41. Schoutens, W.: Lévy Processes in Finance. Wiley, New York (2003)

42. Soner, H.M.: Optimal control with state-space constraint. II. SIAM J. Control Optim. 24, 1110-1122 (1986)

43. Tankov, P.: Dependence structure of Lévy processes with applications to risk management. Rapport Interne No. 502, CMAP École Polytechnique, 2003. http://www.cmap.polytechnique.fr/preprint

44. von Petersdorff, T., Schwab, C.: Numerical solution of parabolic equations in high dimensions. Math. Model. Numer. Anal. 38, 93-127 (2004)

45. Winter, C.: Wavelet Galerkin schemes for option pricing in multidimensional Lévy models. Ph.D. Thesis, ETH Zürich, 2009. http://e-collection.ethbib.ethz.ch/eserv/eth:41555/eth-41555-02.pdf 